\title{
Recovery of Cellular Traction in Three-Dimensional Nonlinear Hyperelastic Matrices
}

\author{
Li Dong ${ }^{\mathrm{a}}$, Assad A. Oberai ${ }^{\mathrm{a}, *}$ \\ ${ }^{a}$ Scientific Computation Research Center (SCOREC), Rensselaer Polytechnic Institute, \\ Troy, NY 12180, USA
}

\begin{abstract}
The traction exerted by a cell on the extra-cellular matrix (ECM) is critical to understanding and manipulating important biological processes such as stem cell differentiation, cancer cell metastasis, and embryonic morphogenesis. This traction is typically quantified through traction force microscopy (TFM). In TFM, the displacement of select markers inside the ECM is tracked, and is used in conjunction with an elasticity problem to reconstruct the traction field. Most applications of this technique thus far have assumed that the matrix behaves as a linear elastic solid that undergoes small deformation and infinitesimal strains. In this manuscript, we develop and implement a robust and efficient TFM methodology that overcomes these limitations by accounting for geometric and material nonlinearities in the ECM. We pose the TFM problem as an inverse problem and develop efficient adjoint-based minimization techniques to solve it. We test the effect of measurement noise on the proposed method, and examine the error incurred by not including nonlinear effects when solving the TFM problem. We present these results for in-silico traction fields that are applied to realistic geometric models of microglial and neuronal cells.
\end{abstract}

Keywords: Cell traction force microscopy, Material nonlinearity, Geometric nonlinearity, Stabilized finite element formulation, Inverse problem, Adjoint equations

\footnotetext{
* Corresponding author

Email addresses: dongl3@rpi.edu (Li Dong), oberaa@rpi.edu (Assad A. Oberai)
}

Preprint submitted to Computer Methods in Applied Mechanics and EngineeringMay 10, 2016

(C) 2016. This manuscript version is made available under the Elsevier user license http://www.elsevier.com/open-access/userlicense/1.0/ 


\section{Introduction}

The traction exerted by a cell on its surroundings (and by the surroundings on a cell) plays a critical role in cell development and migration, and is therefore important in understanding and manipulating important biological processes such as stem cell differentiation, cancer cell metastasis, and embryonic morphogenesis [1-3]. In order to quantitatively measure the traction field, several techniques have been developed. These include the use of wrinkling membranes [4, cantilever-based sensing devices [5, 6] and traction force microscopy (TFM) [7 9].

In TFM, the extracellular matrix (ECM) surrounding the cell is embedded with beads, and the motion of these beads in response to cellular tractions is tracked. Given this motion, and the mechanical behavior of the extracellular matrix, the traction at the cell-matrix interface is recovered [10]. When TFM is applied to a thick, two-dimensional, linear-elastic substrate, the problem of determining the tractions is further simplified by utilizing the Boussinesq solution [11] in the Fourier space. This technique is often referred to as Fourier Transform Traction Cytometry (FTTC) 8], and yields a rapid solution for the traction field. However, its application is limited to thick two-dimensional substrates undergoing small strains.

The extension of TFM to three dimensions is considered in [10]. This reference describes an approach to TFM for cells that are geometrically complex and are embedded in a three-dimensional matrix. This problem is solved by constructing a numerical Green's function using the finite element method that connects the traction applied at a point on the cell-matrix interface to the displacement at a given bead location. Once this map is determined, a minimization problem is solved in order to determine the traction field that produces a bead displacement field that best matches the measured displacement field. A similar approach can also be found in [12].

A natural extension to the ideas described above would involve accounting for the nonlinear effects during the elastic deformation of the ECM. There are several sources of this nonlinear behavior:

1. When the strain in a solid exceeds a certain threshold (usually around $10 \%$ ), the linear measure of strain (the infinitesimal strain tensor) is no longer appropriate, and other nonlinear measures that accurately measure the deformation of the material must be adopted [13 15]. We note that in TFM studies, where $30-70 \%$ strains are quite common [10, 16, 17, this level of strain is routinely exceeded. 
2. When the displacement incurred by the solid is large, the change in the current configuration due to this displacement must be accounted for, and this introduces the so-called geometric nonlinearity to the problem.

3. Some materials display a nonlinear stress-strain response, and this leads to a nonlinear constitutive model. This is especially true for materials such as gelatin-agar co-gels [18, that are commonly used as tissuemimicking phantom, or real biological gels [19].

For all these reasons, it is important to consider the nonlinear behavior of the ECM while computing the traction field exerted by the cell on the matrix.

We note that there have been some attempts at solving the 3D nonlinear TFM problem. In [20], the authors consider a nonlinear constitutive model, but do not account for nonlinearities in strain and in the geometrical description of the problem. Moreover, the applicability of their technique appears to be limited to a small range of traction magnitudes, and to a relatively small number of traction beads.

We also note that there are instances adopting a "forward computation method" (described as such by [21]) for determining the cell traction, in contrast to the inverse problem approach described above [9, 22 25]. In the forward method, the strain is obtained by differentiating the experimentally measured displacement field and the stress/traction fields are then determined by applying the constitutive equation. Clearly, the quality of the cellular tractions recovered using this approach depends heavily on the ability to measure the displacement field close to the cell-matrix interface [21]. This places a significant burden on the experimental set up. The inverse problem approach, on the other hand, can make use of all available bead displacement data, including those that are at some distance from the interface.

In this manuscript we present a new approach to solving the nonlinear TFM problem. We pose the problem as an inverse problem, where we are given the displacement of a finite number of beads, and a model for the the mechanical response of the matrix, and we wish to determine the traction field that is consistent with these observations. We solve the inverse problem as a constrained minimization problem, where we seek to find the traction field that yields a displacement field that best matches measured bead displacements. This is done under the constraint that the predicted displacement field satisfies the equations of equilibrium for a nonlinear hyperelastic material. We solve this problem using gradient-based algorithms, and efficiently compute the gradient using an linearized adjoint equation. 
We account for noise by regularizing the recovered traction field through a Tikhonov term. Similar methods for solving inverse problems have been used to determine the spatial distribution of material properties in different fields of mechanics and physics [26 44]. We test the performance of the proposed approach on synthetic data. We create geometric models of cells embedded in a matrix with known material behavior. Thereafter, we apply tractions at the cell-matrix interface and compute the displacement field in the matrix by solving a forward problem. We utilize a portion of the displacement field as the "measured" displacement field, and in some instances, add noise to it. Using this measured displacement in our algorithm, we determine the traction field at the interface. We also utilize this set-up to determine the effect of making the linear elastic assumption while solving the inverse problem, when the actual response of the material is nonlinear. In the inverse problems literature, this error is referred to as model error. We conclude that for some problems the model error can be significant. Moreover, we note that the problem of recovering tractions is not limited to applications in biomechanics, and has also been studied in strucutural dynamics [45-47]. In these studies also, the assumption of linear elasticity is invoked.

The organization of the remainder of this manuscript is as follows: In Section 2, we present the nonlinear forward elasticity problem that describes the response of a three-dimensional hydrogel to cellular traction. In Section 3. we formulate the nonlinear inverse problem to determine the traction field at the cell-matrix interface, given the "experimentally measured" displacements. We also present the essential ingredients of our solution strategy which include a gradient-based minimization algorithm, an adjoint equation, and a special regularization approach. In Section 4, we describe three numerical examples that include tractions exerted on a hydrogel by a microglial cell and a neuronal cell. We use these examples to quantify and understand errors in the reconstructed traction field due to noise as well as incorrect model selection. We conclude with a summary in Section 5.

\section{The forward elasticity problem}

A typical problem of interest consists of a cell embedded within a threedimensional scaffold made from a hydrogel. It is assumed that the mechanical properties of the cell are not known, while that of the gel are known [7, 8, 10, 23]. In this study, the hydrogel around the cell is modeled as an incompressible hyperelastic solid using a modified version of the Blatz (or 
the Veronda-Westmann) model. Treating hydrogel as incompressible is common [7, 8, 10, 23]. In addition, several commonly used hydrogels, such as agar-gelatin co-gels, display significant nonlinear elastic behavior in range of strains encountered in cell-traction studies. For these types of gels the modified Blatz model is a simple constitutive model (with just 2 parameters) that captures the nonlinear stress-strain response.

\subsection{Strong form}

The problem is posed on the domain obtained by subtracting the cell from the hydrogel. Typically, the extent of the hydrogel is too large to fully model in this problem, so the domain is truncated at a certain distance away from the cell where the displacements (due to cellular tractions) have decayed significantly. This truncation is common [10, 20, 48], and the truncation distance usually depends on how fast the traction decays. Authors in [9, 10, 48 have presented some nice illustrations of the decay of tractions.

The displacement field, $\boldsymbol{u}$, and the pressure, $p$, within the hydrogel obey the equations of equilibrium given by:

$$
\begin{aligned}
\nabla \cdot \boldsymbol{P} & =\mathbf{0} \text { in } \Omega_{0}, \\
\boldsymbol{u} & =\boldsymbol{g} \text { on } \Gamma_{\boldsymbol{g}}, \\
\boldsymbol{P} \cdot \boldsymbol{N} & =\boldsymbol{h} \text { on } \Gamma_{\boldsymbol{h}},
\end{aligned}
$$

along with the incompressibility constraint:

$$
J-1=0, \text { in } \Omega_{0} .
$$

Here Eq.(1) is a statement of equilibrium in the reference configuration $\Omega_{0}$, where $\boldsymbol{P}$ is the first Piola-Kirchhoff stress tensor, and Eqs. (2) and (3) define the Dirichlet (displacement) and Neumann (traction) boundary conditions, respectively, where the displacement data, $\boldsymbol{g}$, is prescribed on $\Gamma_{\boldsymbol{g}}$, and the traction data, $\boldsymbol{h}$, is prescribed on $\Gamma_{\boldsymbol{h}}$. Further, $\partial \Omega_{0}=\overline{\Gamma_{\boldsymbol{g}} \cup \Gamma_{\boldsymbol{h}}}$ defines the closed boundary of $\Omega_{0}, \Gamma_{\boldsymbol{g}} \bigcap \Gamma_{\boldsymbol{h}}=\emptyset$, and $\boldsymbol{N}$ is the outward normal vector on the surface, $\Gamma_{\boldsymbol{g}}$, in the reference configuration. Eq. (4) enforces the incompressibility constraint, and $J=\operatorname{det}(\boldsymbol{F})$ is the jacobian of the deformation gradient, which is defined as $\boldsymbol{F}=\nabla \boldsymbol{u}+\mathbf{1}$.

These equations are appended with a constitutive equation for the stress. In particular, first Piola-Kirchhoff stress tensor is given by $\boldsymbol{P}=\boldsymbol{F} \boldsymbol{S}$, where $\boldsymbol{S}$, the second Piola-Kirchhoff stress tensor, is defined as, $\boldsymbol{S}=-J \times p \boldsymbol{F}^{-\mathbf{1}} \boldsymbol{F}^{-\boldsymbol{T}}+$ $2 \frac{\partial W}{\partial \boldsymbol{C}}$. In the expression above, $W$ is the strain energy density function that 
depends on the invariants of the right Cauchy-Green strain tensor, $\boldsymbol{C}=$ $\boldsymbol{F}^{\boldsymbol{T}} \boldsymbol{F}$. For the constitutive model used in this paper, $W$ is given by

$$
W=\frac{\mu}{2 \gamma}\left(e^{\gamma\left(J^{-\frac{2}{3}} I_{1}-3\right)}-1\right),
$$

where $\mu$ is the shear modulus at zero strain, and $\gamma$ is the nonlinear parameter that determines the nonlinear change of stress with strain. Further, $I_{1}=$ trace $(\boldsymbol{C})$, is the first principal invariant of the Cauchy-Green strain tensor. More details about this material model can be found in [34, 49 51].

\subsection{Weak form}

We solve the forward problem using the finite element method, which is based on the weak, or the variational form, of the forward problem. The variational form may be derived by either minimizing the total potential energy of the system under the constraint of incompressibility, or it may be derived by multiplying the equilibrium equation and the incompressibility equations with weighting functions, integrating over $\Omega_{0}$, performing integration-by-parts on the former, and then imposing the traction boundary condition Eq.(3). In either case, we arrive at the following statement: Find $\boldsymbol{U} \equiv[\boldsymbol{u}, p] \in \mathcal{S} \times \mathcal{P}$ such that

$$
\mathcal{A}(\boldsymbol{W}, \boldsymbol{U})=(\boldsymbol{w}, \boldsymbol{h})_{\Gamma_{\boldsymbol{h}}}, \quad \forall \boldsymbol{W} \equiv[\boldsymbol{w}, q] \in \mathcal{V} \times \mathcal{P},
$$

where

$$
\begin{array}{r}
\mathcal{A}(\boldsymbol{W}, \boldsymbol{U}) \equiv \int_{\Omega_{0}} \nabla \boldsymbol{w}: \boldsymbol{P} d \Omega+\int_{\Omega_{0}} q(J-1) d \Omega, \\
(\boldsymbol{w}, \boldsymbol{h})_{\Gamma_{\boldsymbol{h}}} \equiv \int_{\Gamma_{\boldsymbol{h}}} \boldsymbol{w} \cdot \boldsymbol{h} d \Gamma .
\end{array}
$$

The function spaces $\mathcal{V}, \mathcal{S}$, and $\mathcal{P}$ that appear in the equations above are defined as,

$$
\begin{array}{r}
\mathcal{V}=\left\{\boldsymbol{w} \mid w_{i} \in H^{1}\left(\Omega_{0}\right) ; w_{i}=0 \text { on } \Gamma_{\boldsymbol{g}}\right\}, \\
\mathcal{S}=\left\{\boldsymbol{u} \mid u_{i} \in H^{1}\left(\Omega_{0}\right) ; u_{i}=g_{i} \text { on } \Gamma_{\boldsymbol{g}}\right\}, \\
\mathcal{P} \subseteq L_{2}\left(\Omega_{0}\right),
\end{array}
$$

where $L_{2}$ is the space of square-integrable functions, and $H^{1}$ is the Sobolev space of square-integrable functions with square-integrable derivatives. 
The standard Galerkin finite element method is obtained by restricting the weak form (Eq 6 ) to finite-dimensional function spaces. However, for incompressible materials, it is well known that the Galerkin finite element method leads to unstable solutions unless very specific choices are used for the displacement and pressure function spaces. This restriction can be overcome by stabilizing this formulation with residual-based terms [52, 53. With that addition, the discrete stabilized formulation is given by: Find $\boldsymbol{U}^{h} \equiv\left[\boldsymbol{u}^{h}, p^{h}\right] \in$ $\mathcal{S}^{h} \times \mathcal{P}^{h}$ such that

$$
\mathcal{A}_{s}\left(\boldsymbol{W}^{h}, \boldsymbol{U}^{h}\right)=\left(\boldsymbol{w}^{h}, \boldsymbol{h}\right)_{\Gamma_{h}}, \quad \forall \boldsymbol{W}^{h} \equiv\left[\boldsymbol{w}^{h}, q^{h}\right] \in \mathcal{V}^{h} \times \mathcal{P}^{h},
$$

where

$$
\mathcal{A}_{s}\left(\boldsymbol{W}^{h}, \boldsymbol{U}^{h}\right) \equiv \mathcal{A}\left(\boldsymbol{W}^{h}, \boldsymbol{U}^{h}\right)-\sum_{e=1}^{n_{e l}}\left(\tau \nabla \cdot\left(\boldsymbol{F}^{h} \boldsymbol{S}^{h}\right), \boldsymbol{F}^{h-T} \nabla q^{h}\right)_{\Omega_{0}^{e}} .
$$

The second term in Eq 13 represents the stabilization term. The subscript $\Omega_{0}^{e}$ indicates that the $L_{2}$ inner product is evaluated in the interior of the $e$ th element, and the stabilization factor, $\tau=\frac{\alpha h^{2}}{2 \mu}$, where $\alpha \approx 0.5$, and $h$ is the characteristic element length. A detailed discussion of this stabilization method can be found in [34. In our implementation, all variables, including displacement, pressure and traction, are represented by bilinear finite element basis functions.

\section{Inverse problem for the traction vector field}

In this section, we consider the inverse problem of determining the traction field, $\boldsymbol{h}$, on the boundary, $\Gamma_{\boldsymbol{h}}$, given measured displacements at discrete locations in the hydrogel. The overall scheme to obtain the solution of this inverse problem is similar to that of the material property identification inverse problem described in [34] and 29]. The key difference is that we wish to recover the traction, $\boldsymbol{h}$, and not the material properties. The inverse problem is formulated as a minimization problem subject to the constraint of satisfying the forward problem. A gradient-based optimization approach, L-BFGS [54], is used to solve the minimization problem iteratively and the gradients are efficiently evaluated by solving the appropriate adjoint equation [26]. Similar use of the adjoint method to efficiently compute gradients can also be found in other applications [55 58. 


\subsection{Inverse problem statement}

The inverse problem is stated as follows: Given the measured displacement field, $\tilde{\boldsymbol{u}}$, find the traction field, $\boldsymbol{h}$, such that the objective function

$$
\pi(\boldsymbol{u}, \boldsymbol{h})=\frac{1}{2}\|\boldsymbol{T} \boldsymbol{u}-\boldsymbol{T} \tilde{\boldsymbol{u}}\|_{0}^{2}+\alpha \mathcal{R}(\boldsymbol{h})
$$

is minimized subject to the constraint that the predicted displacement field, $\boldsymbol{u}$, satisfies Eqs.(1)-(4).

In Eq. (14), the first term is the displacement matching term. The mismatch between the predicted and measured displacement field is measured in the $L_{2}$ norm denoted by $\|\cdot\|_{0}$. It is weighted by the tensor $\boldsymbol{T}$, which can be used to augment the contributions from the more accurate components of measured displacements. The second term of Eq. (14) is the regularization term, where $\alpha$ is the regularization parameter. The regularization term embeds prior information about the unknown field, in our case the traction field, $\boldsymbol{h}$, into the inverse problem. In most cases, it is assumed that the recovered field is smooth in a sense defined by the choice of this term. This has the effect of regularizing the inverse problem, which is typically ill-posed, as it lacks uniqueness or stability. In this manuscript, Tikhonov $\left(H_{1}\right)$ regularization [59] is implemented:

$$
\mathcal{R}(\boldsymbol{h})=\frac{1}{2} \int_{\Gamma_{\boldsymbol{h}}}\left|\nabla_{\Gamma} \boldsymbol{h}\right|^{2} d \Gamma .
$$

In the equations above, $\nabla_{\Gamma}$ represents the gradient operator restricted to the surface $\Gamma_{h}$. At any location $\boldsymbol{x} \in \Gamma_{\boldsymbol{h}}$, it is given by $\nabla_{\Gamma} \equiv(\mathbf{1}-\boldsymbol{N} \otimes \boldsymbol{N}) \nabla$, where $\mathbf{1}$ is the identity tensor, $\boldsymbol{N}$ is the outward normal to the surface and $\nabla$ is the gradient operator. We note that $\nabla_{\Gamma}$ only retains the tangential components of $\nabla$.

\subsection{Formulation of the gradient}

In order to solve the inverse problem using a quasi-Newton method, we need to evaluate the derivative of $\pi$ with respect to $\boldsymbol{h}$. This is relatively easily evaluated for the regularization term. However, its evaluation for the displacement mismatch term is cumbersome. This is because the predicted displacement field, $\boldsymbol{u}$, depends upon $\boldsymbol{h}$ implicitly through the constraint of Eq. 12. This difficulty is circumvented through the use of an appropriately 
chosen adjoint field, which is derived by constructing the Lagrangian corresponding to $\pi$. That is,

$$
L\left(\boldsymbol{U}^{h}, \boldsymbol{W}^{h}, \boldsymbol{h}\right)=\pi\left(\boldsymbol{u}^{h}, \boldsymbol{h}\right)+\mathcal{A}_{s}\left(\boldsymbol{W}^{h}, \boldsymbol{U}^{h}\right)-\left(\boldsymbol{w}^{h}, \boldsymbol{h}\right)_{\Gamma_{\boldsymbol{h}}},
$$

where $\boldsymbol{W}^{h} \in \mathcal{V}^{h} \times \mathcal{P}^{h}$ and $\boldsymbol{U}^{h} \in \mathcal{S}^{h} \times \mathcal{P}^{h}$. Here $\boldsymbol{W}^{h}$ plays the role of a Lagrange multiplier field.

We denote the variation of a function $f(x)$ in the direction $\delta x$ by $\delta f$,

$$
\delta f=D_{x} f \cdot \delta x=\left.\frac{d}{d \epsilon} f(x+\epsilon \delta x)\right|_{\epsilon \rightarrow 0} .
$$

The variation of $L$ is given by

$$
\delta L=D_{\boldsymbol{U}^{h}} L \cdot \delta \boldsymbol{U}^{h}+D_{\boldsymbol{W}^{h}} L \cdot \delta \boldsymbol{W}^{h}+D_{\boldsymbol{h}} L \cdot \delta \boldsymbol{h} .
$$

Setting $D_{\boldsymbol{W}^{h}} L \cdot \delta \boldsymbol{W}^{h}=0, \forall \delta \boldsymbol{W}^{h} \in \mathcal{V}^{h} \times \mathcal{P}^{h}$ in the above equation immediately yields an equation for $\boldsymbol{u}^{h}$, that is

$$
\mathcal{A}_{s}\left(\delta \boldsymbol{W}^{h}, \boldsymbol{U}^{h}\right)=\left(\delta \boldsymbol{w}^{h}, \boldsymbol{h}\right)_{\Gamma_{\boldsymbol{h}}}, \quad \forall \delta \boldsymbol{W}^{h} \in \mathcal{V}^{h} \times \mathcal{P}^{h} .
$$

This implies that $\boldsymbol{U}^{h}$ satisfies the forward problem Eq. 12). Under this condition, from Eq.(16), we conclude $\pi=L$, and hence $\delta \pi=\delta L$.

We now examine the condition for which the expression for $\delta L$ can be further simplified. In particular, we set $D_{\boldsymbol{U}^{h}} L \cdot \delta \boldsymbol{U}^{h}=0, \forall \delta \boldsymbol{U}^{h} \in \mathcal{V}^{h} \times \mathcal{P}^{h}$ to arrive at

$$
\mathcal{B}_{s}\left(\boldsymbol{W}^{h}, \delta \boldsymbol{U}^{h} ; \boldsymbol{U}^{h}\right)=-(\boldsymbol{T} \delta \boldsymbol{u}, \boldsymbol{T} \boldsymbol{u}-\boldsymbol{T} \tilde{\boldsymbol{u}}), \quad \forall \delta \boldsymbol{U}^{h} \in \mathcal{V}^{h} \times \mathcal{P}^{h} .
$$

where $\left.\mathcal{B}_{s}\left(\boldsymbol{W}^{h}, \delta \boldsymbol{U}^{h} ; \boldsymbol{U}^{h}\right) \equiv \frac{d}{d \epsilon} \mathcal{A}_{s}\left(\boldsymbol{W}^{h}, \boldsymbol{U}^{h}+\epsilon \delta \boldsymbol{U}^{h}\right)\right|_{\epsilon \rightarrow 0}$ is the linearization of $\mathcal{A}_{s}(\cdot, \cdot)$ about $\boldsymbol{U}^{h}$. Eq. 20 yields a well-posed linear problem for $\boldsymbol{W}^{h}$. The left hand side of this problem is the adjoint of the linearized version of the original forward problem Eq. (12). For this reason, this problem is referred to as the adjoint problem, and $\boldsymbol{W}^{h}$ the adjoint field.

With $\boldsymbol{U}^{h}$ given by the primal problem Eq. (19) and $\boldsymbol{W}^{h}$ given by the adjoint problem Eq.(20), we have from Eq.(18)

$$
\delta L=\delta \pi=D_{\boldsymbol{h}} L \cdot \delta \boldsymbol{h}=\alpha D_{\boldsymbol{h}} \mathcal{R} \cdot \delta \boldsymbol{h}-\left(\boldsymbol{w}^{h}, \delta \boldsymbol{h}\right)_{\Gamma_{\boldsymbol{h}}},
$$

where $\mathcal{R}$ is the regularization term defined by Eq. (15). As a result, the algorithm to calculate the change in the objective function corresponding to a change in the traction vector proceeds as follows: 
1. Solve Eq. 19 to determine the primal field $\boldsymbol{U}^{h}=\left[\boldsymbol{u}^{h}, p^{h}\right]$.

2. Solve Eq. 20$)$ to determine the adjoint field $\boldsymbol{W}^{h}=\left[\boldsymbol{w}^{h}, q^{h}\right]$.

3. Use $\boldsymbol{w}^{h}$ in Eq. (21) to determine the variation in the objective function.

In this study, the optimization variables are the traction components at each node on the cell-matrix interface. The value of the objective function, Eq.(14), and its gradient, Eq.(21), are the input for the L-BFGS optimization algorithm, which returns an updated estimate of tractions for each node and the whole process is repeated until convergence or until the maximum number of iterations is achieved.

\section{Remark}

We note that the primal problem, Eq.(19), is a system of nonlinear equations which is solved using the Newton method. This represents the major computational cost of our algorithm. This cost is kept in check by utilizing a continuation scheme described in [34] and [29]. Within this approach, when solving the forward traction problem for a new guess of the traction field, the solution from the previous step is used as an initial state. Consequently, the forward problem is solved within 10 Newton iterations, as opposed to on the order of 100 iterations otherwise.

\section{Numerical examples}

In this section, two geometrically accurate cell models are used to demonstrate the applicability of our nonlinear algorithm in recovering cell traction. In each case, a known traction field, $\hat{\boldsymbol{h}}$, is applied at the cell-matrix interface and the forward problem is solved. This results in the displacement of the matrix which is sampled at some select nodes. We denote this displacement vector by $\hat{\boldsymbol{u}}^{(j)}, j=1, \cdots, n_{\text {node }}$. In some cases, in order to simulate the effect of instrument noise and errors in the displacement estimation algorithm in actual experiments, noise is added to this displacement. The noisy "measured" displacement field is given by

$$
\tilde{\boldsymbol{u}}^{(i)}=\hat{\boldsymbol{u}}^{(i)}+\eta \times \frac{\sum_{j=1}^{n_{\text {node }}}\left|\hat{\boldsymbol{u}}^{(j)}\right|}{\sum_{j=1}^{n_{\text {node }}}\left|\boldsymbol{n}^{(j)}\right|} \times \boldsymbol{n}^{(i)}, i=1, \cdots, n_{\text {node }}
$$

where $|\cdot|$ denotes the magnitude of a vector, $n_{k}^{(j)} \in N(0,1), \quad k=1,2,3$ and $N(0,1)$ is standard normal distribution. In this case, $\eta$, represents the 
fraction of noise in the "measured" displacement field. The effect of this parameter on the quality of the reconstruction is examined.

In order to quantify the performance of our algorithm, the difference between the recovered traction field, $\boldsymbol{h}$, and the applied traction field, $\hat{\boldsymbol{h}}$, is measured,

$$
e(\boldsymbol{h})=\frac{\|\boldsymbol{h}-\hat{\boldsymbol{h}}\|_{0}}{\|\hat{\boldsymbol{h}}\|_{0}} .
$$

For simplicity, a weight of unity is selected for the entire displacement vector, which means that the tensor, $\boldsymbol{T}$, in Eq.(14) is set to the identity tensor. In order to be unbiased, the initial guess for the traction field is set to 0 . Also, the regularization parameter for the following results in this paper was obtained by solving the inverse problem with different values of the regularization parameter, and plotting the variation of displacement mismatch term as a function of the regularization parameter (the so-called L-curve [60]). See Figure 4, for the L-curve for a typical case considered in this study. The quasi-Newton iterations were considered "converged" when the relative change in the displacement matching term over the last five iterations was smaller than $1 \times 10^{-8}$.

\subsection{Microglial cell model}

The 3D model of the microglial cell (see Figure 1a) is reconstructed through a set of pictures of z-stack microscopy (image courtesy of Sebastian Rhode at Carl Zeiss Microscopy and Sabine Scheibe) with ImageJ [61] and meshed with Simmodeler (Simmertrix.Inc, New York, US). The cell is about $20 \mu \mathrm{m} \times 20 \mu \mathrm{m} \times 15 \mu \mathrm{m}$ and is contained within a cubical matrix with an edge length of $100 \mu \mathrm{m}$. The finite element mesh is comprised of 9,641 vertices and 51,544 tetrahedal elements. Out of these, the vertices that are on the surface of the cell, and beyond a sphere of $50 \mu \mathrm{m}$ diameter, are treated as location without beads. That is the displacement field from these locations is not used in solving the inverse problem. This gives us a total of 7,023 bead locations to work with. The exclusion of other locations helps us in avoiding the "inverse crime" of using all the forward data in the inverse problem. It is also consistent with the experimental protocol where most of the beads that are close to the cell are tracked [62]. We note that the number of beads considered in this study is comparable to experiments [10] and is much more than some of the state-of-the-art cell traction recovery algorithms can handle [12, 20]. 
In the numerical experiments reported here, the shear modulus, $\mu$, of the matrix is set to either $400 \mathrm{~Pa}$ or $600 \mathrm{~Pa}$. The values lie within the range of commonly used experimental hydrogels, extracellular matrices and real tissues [10, 63, 64]. The dimensionless nonlinear parameter, $\gamma$, in the modified blatz model is set to either 1 or 5 . With $\gamma=1$ the material behaves linearly up to about $100 \%$ strain, and with $\gamma=5$ it starts to deviate from linear behavior at about $15 \%$ strain [34]. All six surfaces of the box are assumed fixed in the $x, y$ and $z$ directions $\left(u_{x}=u_{y}=u_{z}=0\right)$.

As shown in Figure 1b, the traction field is applied such that it is maximum along the two protrusions of the cell, and is smoothly interpolated between these regions. The direction of the traction field is roughly pointed to the center of the cell. This loading condition simulates the contraction of the matrix close to the cell after the cell is lysed with detergent [10].

In the following sections, we consider the effect of material and geometric nonlinearity in recovering the traction vector field. In the first section, we set $\gamma=1$ so that within the range of strains considered, the matrix behaves like a linear elastic solid. Thereafter, we consider $\gamma=5$, where the material displays a nonlinear elastic response. We note that while gelatin substrates tend to be linear elastic for large values of strain, gelatin-agar substrates display nonlinear elastic behavior at relatively small $(\approx 15 \%)$ strains $[18$.

\section{Linear material $(\gamma=1)$}

The shear modulus for the matrix is set to $400 \mathrm{~Pa}$. In Figure 1b, we observe that the largest tractions are applied at the tips of the cell, and the magnitude drops as we approach the center of the cell. The traction pulls each arm of the cell toward the center of the cell. The resulting displacement fields are maximum at the tip of the arms, and at the connections between arms and the rest of the cell. Significant strains are generated on the cellmatrix interface, and are illustrated in Figures $1 \mathrm{c}$, where we have plotted the first principal component of the Green-Lagrange strain tensor. We note that this strain ranges from about $50 \%$ to $90 \%$ on the arms - Figure $1 \mathrm{~d}$. The large strain indicates that the use of a linear measure of strain is inappropriate for this problem.

For each set of "measured" displacements (with $1 \%$ and without noise), the traction field was reconstructed using either a finite strain or an infinitesimal strain model. The resulting traction vector fields are shown in Figure 2. We observe that while the spatial variation of these fields is very similar, their magnitudes are significantly different. This is made clear by computing 
the error, $e(\boldsymbol{h})$, in the reconstructed traction field (see Figure 3). For the no-noise case with the finite strain model, this error is $4.23 \%$, and with the infinitesimal strain model it is $12.7 \%$ (regularization parameters $=0.0001$ and 1 , respectively). When $1 \%$ noise is added to the displacements, the error is $12.75 \%$ for the finite strain model, and $20.7 \%$ for the infinitesimal strain model (regularization parameters $=5$ and 10, respectively). The difference between these two errors, which is around $8 \%$, is therefore the error incurred in incorrectly making the infinitesimal strain assumption. Here we note that the infinitesimal strain assumption is often made [7-10, 12, 20]. Figure 4 displays the L-curve for the case with finite deformation and $1 \%$ noise as a typical curve for the cases considered in this paper. According to [60], the value of the regularization parameters at the point of largest curvature is the "optimal" regularization parameter. For the example considered in this curve, this corresponds to $\alpha=5$ (circled in blue in Figure 4). We use this approach to find the regularization parameters for all cases in this paper. For the sake of brevity, we do not show all the L-curve plots.

Nonlinear material $(\gamma=5)$

In this case when solving the forward problem, in order to generate the "measured" data, the shear modulus for the matrix is set to $600 \mathrm{~Pa}$, and the nonlinear parameter is set to $\gamma=5$. The traction field is identical to the $\gamma=1$ case described above. The traction field and the displacement magnitude are shown in Figure 5a. We observe that when compared with the linear elastic case, the displacement for this case is smaller. In examining the image for the principal component of the Green-Lagrange strain, we observe that the maximum strain is also much smaller (approximately $40 \%$ as opposed to $90 \%$ ). This is attributed to the higher shear modulus and the larger values of the nonlinear parameter in this case. This leads to a large value of the tangent modulus for the matrix, particularly at large values of strain. Given that the traction vector field for the two cases is the same, a larger elastic tangent modulus implies a small overall strain.

Once again two cases were considered: one with no noise and one with $1 \%$ noise. For each case, two inverse problems were solved. In one problem, the matrix deformation was treated with a finite strain theory and the appropriate nonlinear elastic model was utilized. In the second problem, the deformation was incorrectly treated as linear elastic, and an infinitesimal strain model was used. The resulting traction vectors are shown in Figure 6. The spatial distribution of these fields is very close to the "exact" trac- 
tion field, however their magnitudes are different. Their departures from the applied traction field are quantified in Figure 3. In the no-noise case, the errors, when using the finite strain and infinitesimal strain models, are $4.5 \%$ and $14.76 \%$, respectively (regularization parameter $=0.0001$ and 1 , respectively). The difference between the two is significant. For the $1 \%$ noise case, the corresponding errors are $18.66 \%$ and $27.23 \%$ (regularization parameter $=5$ and 10 , respectively), and the difference is around $9 \%$. Once again we observe that the model error, that is the error associated with assuming an incorrect model in the inverse problem, is significant.

\subsection{Neuronal cell model}

As the second example, the geometry of a multi-polar neuronal cell is considered (see Figure 7a). Recently, there has been significant interest in understanding the traction exerted by these cells [65, 66]. The size of the neuronal cell is about $10 \mu \mathrm{m} \times 10 \mu \mathrm{m} \times 10 \mu \mathrm{m}$, and it is contained within a cube of length $80 \mu \mathrm{m}$. The unstructured mesh for this configuration consists of 54,587 elements with 10,673 vertices. Out of these, 6,971 are treated as tracked beads (that is the displacement at these locations are "measured"). These locations are selected so that they are close to the cell-matrix interface (but not on it). The shear modulus of the surrounding matrix is $400 \mathrm{~Pa}$, and the nonlinear parameter is set to either 1 or 5 . Five of the outer surfaces of the box are fixed in the $x, y$ and $z$ directions $\left(u_{x}=u_{y}=u_{z}=0\right)$, and the sixth surface is traction free, which is to mimic the free top surface of the matrix in experiments.

As shown in Figure $7 \mathrm{~b}$, the traction field is selected such that its magnitude is largest along the arms of the cell and smoothly reduces towards the center. In addition, the traction vector is pointed inward toward the center of the cell. Similar to the first example, this loading condition also simulates the contraction of the matrix close to the cell after the cell is lysed with detergent [10].

\section{Linear material $(\gamma=1)$}

In Figure 7c, we observe that the largest strain occurs at the dendritic ends of the cell, and pulls the dendrites toward the center of the cell body. Significant strains (50\% to $90 \%$ along the dendrites) are generated at the cell-matrix surface, and are illustrated in Figure $7 \mathrm{~d}$, where we have plotted the first principal component of the Green-Lagrange strain tensor. 
When solving the inverse problem, we use displacements with no noise and $1 \%$ noise, and then either use a finite strain or an infinitesimal strain model. The recovered traction vector fields appear similar, and are shown in Figure 8. However, their magnitudes are different. In Figure 9, we quantify this by evaluating the error $(e(\boldsymbol{h}))$ in the traction field for each case. For "measured" displacement with no noise, this error is $0.5 \%$ for the finite strain model, and $16.93 \%$ for the infinitesimal strain model (regularization parameters $=1 \times$ $10^{-7}$ and 0.1 , respectively). For "measured" displacement with $1 \%$ noise, the error for the finite strain model grows to $26.41 \%$, and that for the infinitesimal strain model is $34.78 \%$ (regularization parameters $=0.5$ and 1 , respectively). The difference between these errors is the penalty associated with invoking the infinitesimal strain assumption.

\section{Nonlinear material $(\gamma=5)$}

The problem described above is repeated with the nonlinear parameter now set to 5. This means that the matrix now stiffens with increasing strain, and given that the traction field is unchanged, it strains less in response to the traction exerted by the cell. This can be seen by examining Figures 10a and $10 \mathrm{~b}$ where we have plotted the displacement magnitude and the principal component of the Green-Lagrange strain tensor on the cell-matrix interface.

Once again displacement fields with no noise and with $1 \%$ noise are considered, and when solving the inverse problem, a finite strain model (with the appropriate nonlinear elastic response) and an infinitesimal strain model (with a linear elastic response) is used. This is done to quantify the effect of noise and modeling assumptions in solving the inverse problem. In all cases, we observe that the spatial distributions of the traction vector is recovered with remarkable accuracy (see Figure 11, regularization parameter $=1 \times 10^{-7}, 0.5,0.1$ and 1 , respectively). However, the magnitude of the traction, and consequently the error in the reconstruction (see Figure 9), is sensitive to both displacement noise, and model selection. When there is no noise, the contribution of the model error is greater than $20 \%$, and with $1 \%$ noise it drops to around $8 \%$.

\subsection{Discussion}

We have selected the microglial and neuorn cells as examples because of their biomedical relevance, and also because of their distinct shapes. While the microglial cell has a relatively smooth shape with very few protrusions, the shape of the neuron is much more complicated with several dendritic 
structures. We note that in both cases our algorithm could be applied successfully. However, for the no-noise cases the tractions for the neuron cell were recovered with almost no error, whereas for the microglial cell they were recovered to around $4 \%$ error. There are several possible explanations for this difference. First, the magnitude of tractions applied to the microglial cell is about two times that of the tractions applied to the neuron, and in both cases the inverse problem begins with a zero initial guess. Consequently, for the microglial cell quasi-Newton algorithm has to cover a larger range in order to achieve the exact value. Also the dendritic shape of the neuron implies that there are many more local regions where the strains are large and these regions are sampled by the tracking beads. This could imply that the measured displacement field for the neuron carries a "stronger" imprint of the traction field making the inverse problem better posed.

In biomechanics applications, often the tractions measured are used to determine average quantities such as the contractile moment of the cell, or the net strain energy in the gel. We may conjecture how the proposed method would perform in evaluating these quantities. We note that the contractile moment is a linear functional of the traction field. Thus we would expect that error observed in this quantity would be similar to the error observed in the traction field. On the other hand, the strain energy in the gel may be evaluated by computing the work done by the cell on the gel. That is the integral $\int_{\Gamma} \boldsymbol{h} \cdot \boldsymbol{u} d \Gamma$. In this integral, $\boldsymbol{h}$ is the reconstructed traction field, and $\boldsymbol{u}$ is the estimated displacement on the interface. It is reasonable to expect that if the recovered traction is underestimated by a certain fraction, then the recovered displacement will also be underestimated by the same factor. Then roughly speaking, the integral, which involves the product of these quantities, will be underestimated by two times this factor. In this case the importance of including nonlinear elastic effects will be even more significant.

\section{Summary}

Traction force microscopy is an important technique that is used to quantify tractions at the cell-matrix interface. It works by measuring the motion of embedded micro-beads in response to cellular tractions and using this information to infer the traction field. In this manuscript we have posed TFM as an inverse problem, and solved it using a constrained minimization algorithm. In doing so we have accounted for the three-dimensional 
nature of the problem, the complex geometry of the cell, and the finitestrain and nonlinear elastic behavior of the matrix. Through the use of a quasi-Newton algorithm, a carefully derived adjoint problem, and a novel continuation strategy, we have developed and implemented algorithms that efficiently utilize data from a large number of tracking beads $\left(\approx 10^{4}\right)$ and reconstruct traction vectors on a well-resolved surface mesh $\left(\approx 10^{3}-10^{4}\right.$ grid points).

We have applied these algorithms to in-silico problems with realistic geometric models of microglial and neuronal cells. We conclude that the proposed algorithms are able to accurately recover the traction fields. We have also tested the effect of measurement noise on the proposed methods, and examined the error incurred by not including nonlinear effects when solving the TFM problem. We conclude that the error in the recovered traction field is sensitive to noise in the measured displacements, and to neglecting nonlinear effects in the TFM problem.

\section{Acknowledgement}

The authors acknowledge the support of the NSF grant \#1148,111. 


\section{References}

[1] A. J. Engler, S. Sen, H. L. Sweeney, D. E. Discher, Matrix elasticity directs stem cell lineage specification, Cell 126 (4) (2006) 677-689.

[2] P. Friedl, K. Wolf, Tumour-cell invasion and migration: diversity and escape mechanisms, Nature Reviews Cancer 3 (5) (2003) 362-374.

[3] D. A. Lauffenburger, A. F. Horwitz, Cell migration: a physically integrated molecular process, Cell 84 (3) (1996) 359-369.

[4] A. K. Harris, P. Wild, D. Stopak, Silicone rubber substrata: a new wrinkle in the study of cell locomotion, Science 208 (4440) (1980) 177179.

[5] C. G. Galbraith, M. P. Sheetz, A micromachined device provides a new bend on fibroblast traction forces, Proceedings of the National Academy of Sciences 94 (17) (1997) 9114-9118.

[6] J. L. Tan, J. Tien, D. M. Pirone, D. S. Gray, K. Bhadriraju, C. S. Chen, Cells lying on a bed of microneedles: an approach to isolate mechanical force, Proceedings of the National Academy of Sciences 100 (4) (2003) 1484-1489.

[7] M. Dembo, Y.-L. Wang, Stresses at the cell-to-substrate interface during locomotion of fibroblasts, Biophysical journal 76 (4) (1999) 2307-2316.

[8] J. P. Butler, I. M. Tolić-Nørrelykke, B. Fabry, J. J. Fredberg, Traction fields, moments, and strain energy that cells exert on their surroundings, American Journal of Physiology-Cell Physiology 282 (3) (2002) C595C605.

[9] Z. Yang, J.-S. Lin, J. Chen, J. H. Wang, Determining substrate displacement and cell traction fieldsa new approach, Journal of theoretical biology 242 (3) (2006) 607-616.

[10] W. R. Legant, J. S. Miller, B. L. Blakely, D. M. Cohen, G. M. Genin, C. S. Chen, Measurement of mechanical tractions exerted by cells in three-dimensional matrices, Nature methods 7 (12) (2010) 969-971.

[11] L. D. Landau, E. M. Lifshits, J. B. Sykes, W. H. Reid, Course of theoretical physics: theory of elasticity, Butterworth-Heinemann, 1986. 
[12] G. Vitale, L. Preziosi, D. Ambrosi, A numerical method for the inverse problem of cell traction in 3d, Inverse Problems 28 (9) (2012) 095013.

[13] M. E. Gurtin, An introduction to continuum mechanics, Academic press, 1982.

[14] M. E. Gurtin, E. Fried, L. Anand, The mechanics and thermodynamics of continua, Cambridge University Press, 2010.

[15] Y.-c. Fung, A first course in continuum mechanics, Englewood Cliffs, NJ, Prentice-Hall, Inc., 1977. 351 p. 1.

[16] T. Boudou, J. Ohayon, C. Picart, R. I. Pettigrew, P. Tracqui, Nonlinear elastic properties of polyacrylamide gels: implications for quantification of cellular forces, Biorheology 46 (3) (2009) 191-205.

[17] J. W. Stone, P. N. Sisco, E. C. Goldsmith, S. C. Baxter, C. J. Murphy, Using gold nanorods to probe cell-induced collagen deformation, Nano letters 7 (1) (2007) 116-119.

[18] T. Z. Pavan, E. L. Madsen, G. R. Frank, A. A. O. Carneiro, T. J. Hall, Nonlinear elastic behavior of phantom materials for elastography, Physics in medicine and biology 55 (9) (2010) 2679.

[19] C. Storm, J. J. Pastore, F. C. MacKintosh, T. C. Lubensky, P. A. Janmey, Nonlinear elasticity in biological gels, Nature 435 (7039) (2005) 191-194.

[20] J. Palacio, A. Jorge-Peñas, A. Muñoz-Barrutia, C. Ortiz-de Solorzano, E. de Juan-Pardo, J. García-Aznar, Numerical estimation of 3d mechanical forces exerted by cells on non-linear materials, Journal of biomechanics 46 (1) (2013) 50-55.

[21] M. S. Hall, R. Long, X. Feng, Y. Huang, C.-Y. Hui, M. Wu, Toward single cell traction microscopy within 3 d collagen matrices, Experimental cell research 319 (16) (2013) 2396-2408.

[22] S. A. Maskarinec, C. Franck, D. A. Tirrell, G. Ravichandran, Quantifying cellular traction forces in three dimensions, Proceedings of the National Academy of Sciences 106 (52) (2009) 22108-22113. 
[23] S. S. Ng, C. Li, V. Chan, Experimental and numerical determination of cellular traction force on polymeric hydrogels, Interface focus 1 (5) (2011) 777-791.

[24] N. Gjorevski, C. M. Nelson, Mapping of mechanical strains and stresses around quiescent engineered three-dimensional epithelial tissues, Biophysical journal 103 (1) (2012) 152-162.

[25] T. M. Koch, S. Münster, N. Bonakdar, J. P. Butler, B. Fabry, et al., 3d traction forces in cancer cell invasion, PLoS One 7 (3) (2012) e33476.

[26] A. A. Oberai, N. H. Gokhale, G. R. Feijóo, Solution of inverse problems in elasticity imaging using the adjoint method, Inverse Problems 19 (2) (2003) 297.

[27] A. A. Oberai, N. H. Gokhale, M. M. Doyley, J. C. Bamber, Evaluation of the adjoint equation based algorithm for elasticity imaging, Physics in Medicine and Biology 49 (13) (2004) 2955.

[28] P. E. Barbone, A. A. Oberai, Elastic modulus imaging: some exact solutions of the compressible elastography inverse problem, Physics in medicine and biology 52 (6) (2007) 1577.

[29] N. H. Gokhale, P. E. Barbone, A. A. Oberai, Solution of the nonlinear elasticity imaging inverse problem: the compressible case, Inverse Problems 24 (4) (2008) 045010.

[30] A. A. Oberai, N. H. Gokhale, S. Goenezen, P. E. Barbone, T. J. Hall, A. M. Sommer, J. Jiang, Linear and nonlinear elasticity imaging of soft tissue in vivo: demonstration of feasibility, Physics in medicine and biology 54 (5) (2009) 1191.

[31] M. S. Richards, P. E. Barbone, A. A. Oberai, Quantitative threedimensional elasticity imaging from quasi-static deformation: a phantom study, Physics in medicine and biology 54 (3) (2009) 757.

[32] P. E. Barbone, C. E. Rivas, I. Harari, U. Albocher, A. A. Oberai, Y. Zhang, Adjoint-weighted variational formulation for the direct solution of inverse problems of general linear elasticity with full interior data, International journal for numerical methods in engineering 81 (13) (2010) 1713-1736. 
[33] P. E. Barbone, A. A. Oberai, A review of the mathematical and computational foundations of biomechanical imaging, in: Computational Modeling in Biomechanics, Springer, 2010, pp. 375-408.

[34] S. Goenezen, P. Barbone, A. A. Oberai, Solution of the nonlinear elasticity imaging inverse problem: The incompressible case, Computer methods in applied mechanics and engineering 200 (13) (2011) 1406-1420.

[35] T. J. Hall, P. Barbone, A. A. Oberai, J. Jiang, J. F. Dord, S. Goenezen, T. G. Fisher, Recent results in nonlinear strain and modulus imaging, Current medical imaging reviews 7 (4) (2011) 313.

[36] S. Goenezen, J.-F. Dord, Z. Sink, P. E. Barbone, J. Jiang, T. J. Hall, A. A. Oberai, Linear and nonlinear elastic modulus imaging: an application to breast cancer diagnosis, Medical Imaging, IEEE Transactions on 31 (8) (2012) 1628-1637.

[37] M. Tyagi, S. Goenezen, P. E. Barbone, A. A. Oberai, Algorithms for quantitative quasi-static elasticity imaging using force data, International journal for numerical methods in biomedical engineering 30 (12) (2014) 1421-1436.

[38] U. Albocher, P. E. Barbone, A. A. Oberai, I. Harari, Uniqueness of inverse problems of isotropic incompressible three-dimensional elasticity, Journal of the Mechanics and Physics of Solids 73 (2014) 55-68.

[39] E. P. Canović, D. T. Seidl, S. R. Polio, A. A. Oberai, P. E. Barbone, D. Stamenović, M. L. Smith, Biomechanical imaging of cell stiffness and prestress with subcellular resolution, Biomechanics and modeling in mechanobiology 13 (3) (2014) 665-678.

[40] T. Liu, O. A. Babaniyi, T. J. Hall, P. E. Barbone, A. A. Oberai, Noninvasive in-vivo quantification of mechanical heterogeneity of invasive breast carcinomas, PloS one 10 (7).

[41] J. Worthen, G. Stadler, N. Petra, M. Gurnis, O. Ghattas, Towards adjoint-based inversion for rheological parameters in nonlinear viscous mantle flow, Physics of the Earth and Planetary Interiors 234 (2014) $23-34$. 
[42] A. Gholami, A. Mang, G. Biros, An inverse problem formulation for parameter estimation of a reaction-diffusion model of low grade gliomas, Journal of mathematical biology (2015) 1-25.

[43] M. Aguiló, W. Aquino, J. C. Brigham, M. Fatemi, et al., An inverse problem approach for elasticity imaging through vibroacoustics, Medical Imaging, IEEE Transactions on 29 (4) (2010) 1012-1021.

[44] M. Gockenbach, B. Jadamba, A. Khan, Equation error approach for elliptic inverse problems with an application to the identification of lamé parameters, Inverse Problems in Science and Engineering 16 (3) (2008) 349-367.

[45] D. Schnur, N. Zabaras, Finite element solution of two-dimensional inverse elastic problems using spatial smoothing, International Journal for Numerical Methods in Engineering 30 (1) (1990) 57-75.

[46] L. M. Bezerra, S. Saigal, Inverse boundary traction reconstruction with the bem, International journal of solids and structures 32 (10) (1995) $1417-1431$.

[47] K. Kamiyama, K. Vlack, T. Mizota, H. Kajimoto, N. Kawakami, S. Tachi, Vision-based sensor for real-time measuring of surface traction fields, Computer Graphics and Applications, IEEE 25 (1) (2005) 68-75.

[48] C. Franck, S. A. Maskarinec, D. A. Tirrell, G. Ravichandran, Threedimensional traction force microscopy: a new tool for quantifying cellmatrix interactions, PloS one 6 (3) (2011) e17833.

[49] P. J. Blatz, B. M. Chu, H. Wayland, On the mechanical behavior of elastic animal tissue, Transactions of The Society of Rheology (19571977) 13 (1) (1969) 83-102.

[50] D. Veronda, R. Westmann, Mechanical characterization of skinfinite deformations, Journal of biomechanics 3 (1) (1970) 111-124.

[51] Y. Fung, Elasticity of soft tissues in simple elongation, American Journal of Physiology-Legacy Content 213 (6) (1967) 1532-1544. 
[52] O. Klaas, A. Maniatty, M. S. Shephard, A stabilized mixed finite element method for finite elasticity.: Formulation for linear displacement and pressure interpolation, Computer Methods in Applied Mechanics and Engineering 180 (1) (1999) 65-79.

[53] A. M. Maniatty, Y. Liu, O. Klaas, M. S. Shephard, Higher order stabilized finite element method for hyperelastic finite deformation, Computer Methods in Applied Mechanics and Engineering 191 (13) (2002) 1491-1503.

[54] R. H. Byrd, P. Lu, J. Nocedal, C. Zhu, A limited memory algorithm for bound constrained optimization, SIAM Journal on Scientific Computing 16 (5) (1995) 1190-1208.

[55] D. A. Tortorelli, Sensitivity analysis for non-linear constrained elastostatic systems, International journal for numerical methods in engineering 33 (8) (1992) 1643-1660.

[56] A. Sei, W. W. Symes, et al., Gradient calculation of the traveltime cost function without ray tracing, in: 65th Ann. Internat. Mtg., Soc. Expl. Geophys., Expanded Abstracts, Citeseer, 1994, pp. 1351-1354.

[57] O. Dorn, H. Bertete-Aguirre, J. Berryman, G. Papanicolaou, A nonlinear inversion method for 3d electromagnetic imaging using adjoint fields, Inverse problems 15 (6) (1999) 1523.

[58] G. R. Feijóo, A. A. Oberai, P. M. Pinsky, An application of shape optimization in the solution of inverse acoustic scattering problems, Inverse problems 20 (1) (2004) 199.

[59] A. N. Tikhonov, V. I. Arsenin, F. John, Solutions of ill-posed problems, Winston Washington, DC, 1977.

[60] P. C. Hansen, Analysis of discrete ill-posed problems by means of the l-curve, SIAM review 34 (4) (1992) 561-580.

[61] C. A. Schneider, W. S. Rasband, K. W. Eliceiri, Nih image to imagej: 25 years of image analysis, Nature methods 9 (7) (2012) 671-675.

[62] J. H. Wang, J.-S. Lin, Cell traction force and measurement methods, Biomechanics and modeling in mechanobiology 6 (6) (2007) 361-371. 
698

[63] M. J. Paszek, N. Zahir, K. R. Johnson, J. N. Lakins, G. I. Rozenberg, A. Gefen, C. A. Reinhart-King, S. S. Margulies, M. Dembo, D. Boettiger, et al., Tensional homeostasis and the malignant phenotype, Cancer cell 8 (3) (2005) 241-254.

[64] D. E. Discher, P. Janmey, Y.-l. Wang, Tissue cells feel and respond to the stiffness of their substrate, Science (2005) 1139-1143.

[65] P. C. Kerstein, I. Nichol, H. Robert, T. M. Gomez, Mechanochemical regulation of growth cone motility, Frontiers in Cellular Neuroscience 9 (2015) 244.

[66] J. Jiang, Z.-h. Zhang, X.-b. Yuan, M.-m. Poo, Spatiotemporal dynamics of traction forces show three contraction centers in migratory neurons, The Journal of cell biology 209 (5) (2015) 759-774. 


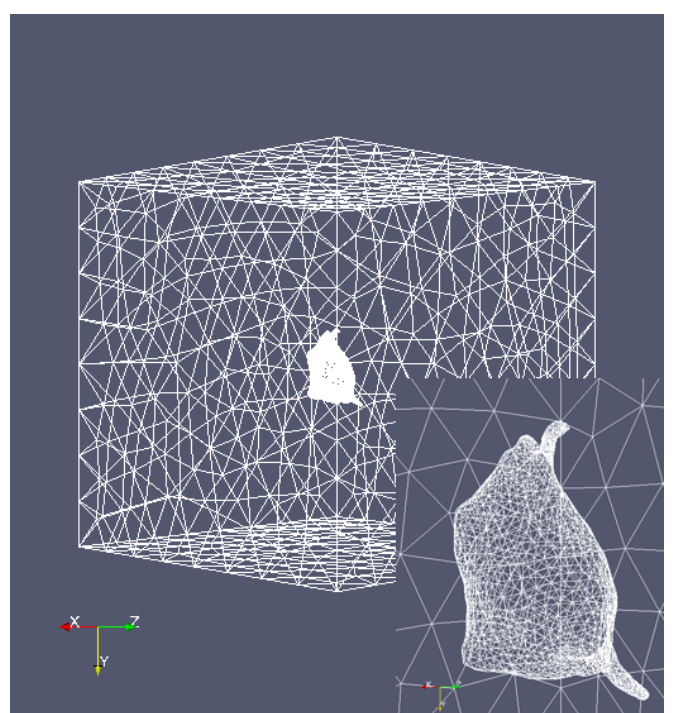

(a)

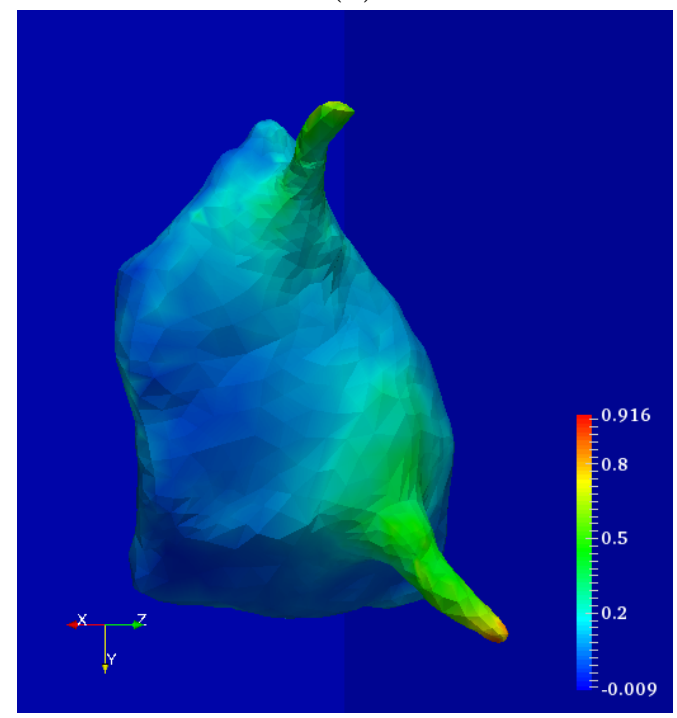

(c)

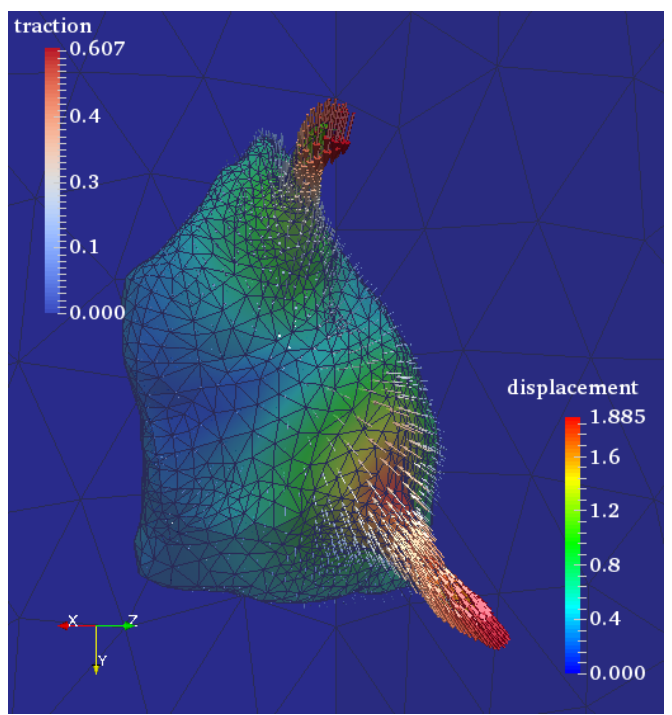

(b)

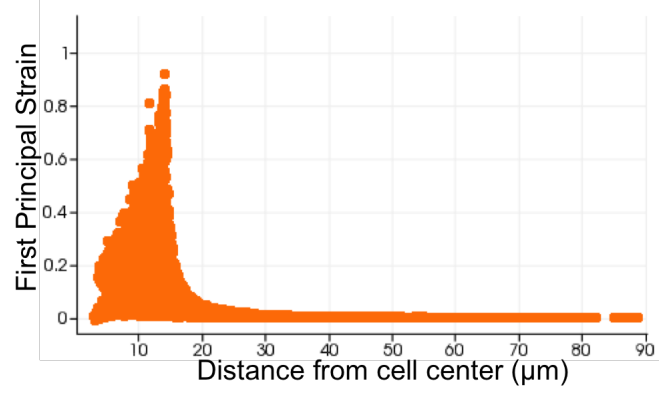

(d)

Figure 1: Microgial cell: (a) Mesh on the exterior surface and the cell-matrix interface. Results of the simulated forward problem on the deformed surface with $\gamma=1$. (b) Arrows display the traction field (in $\mathrm{kPa}$ ), and the deformed surface is colored by the displacement magnitude in $\mu \mathrm{m}$. (c) The first principal component of the Green-Lagrange strain tensor. (d) Distribution of the strain at the bead locations as a function of distance from the center. 


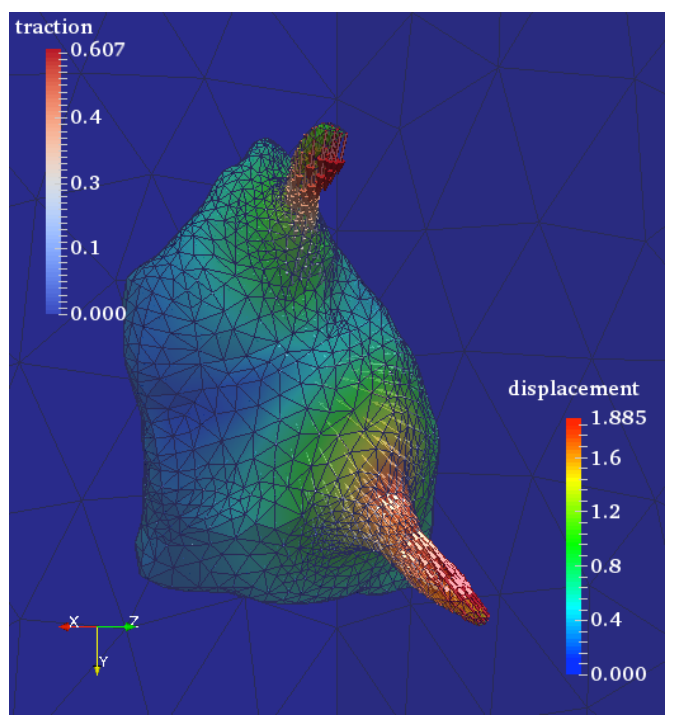

(a)

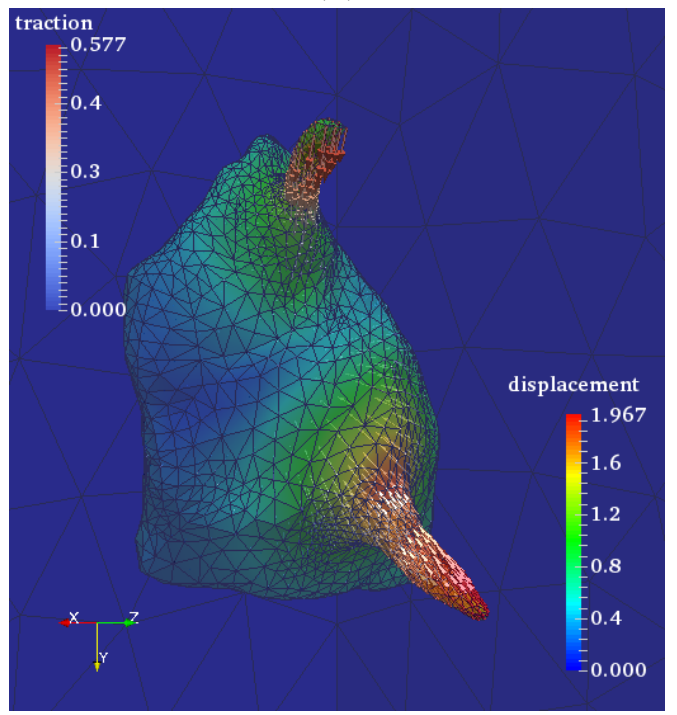

(c)

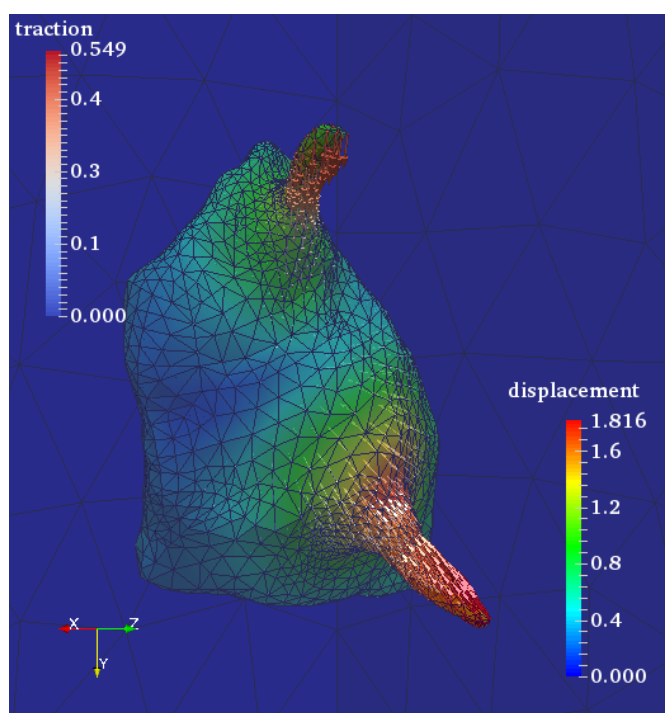

(b)

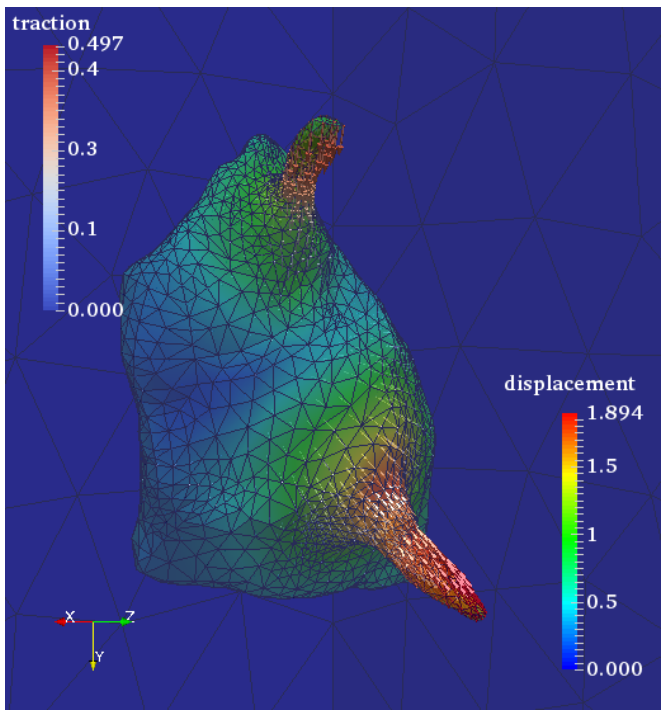

$(\mathrm{d})$

Figure 2: Results of the inverse analysis for the microglial cell on the deformed surface with $\gamma=1$. Arrows display the recovered traction field (in $\mathrm{kPa}$ ), and the deformed surface is colored by the recovered displacement magnitude in $\mu \mathrm{m}$. (a) Solution with nonlinear effects and $0 \%$ noise. (b) Solution with nonlinear effects and $1 \%$ noise. (c) Solution without nonlinear effects and $0 \%$ noise. (d) Solution without nonlinear effects and $1 \%$ noise. 


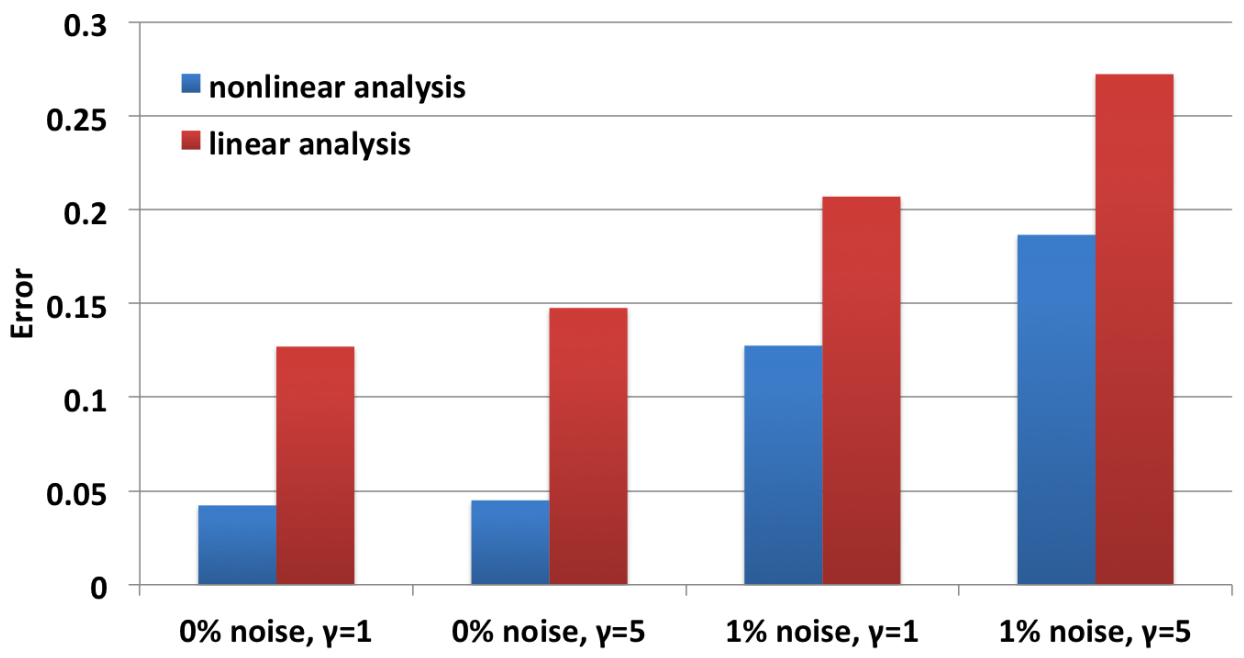

Figure 3: Error summary for the microglial cell model.

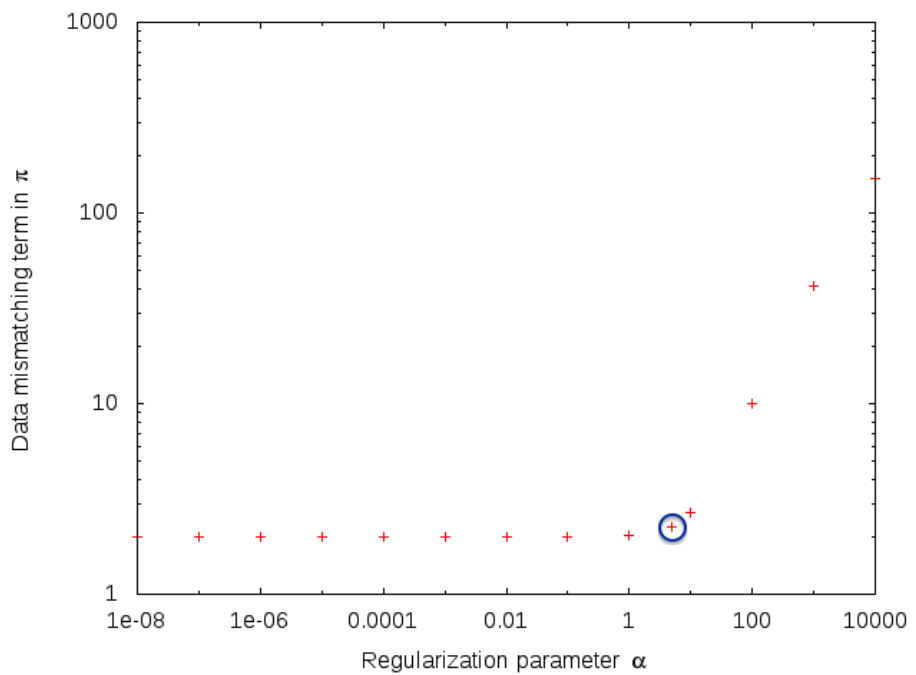

Figure 4: L-curve for the microglial cell model with nonlinear effects and $1 \%$ noise. 


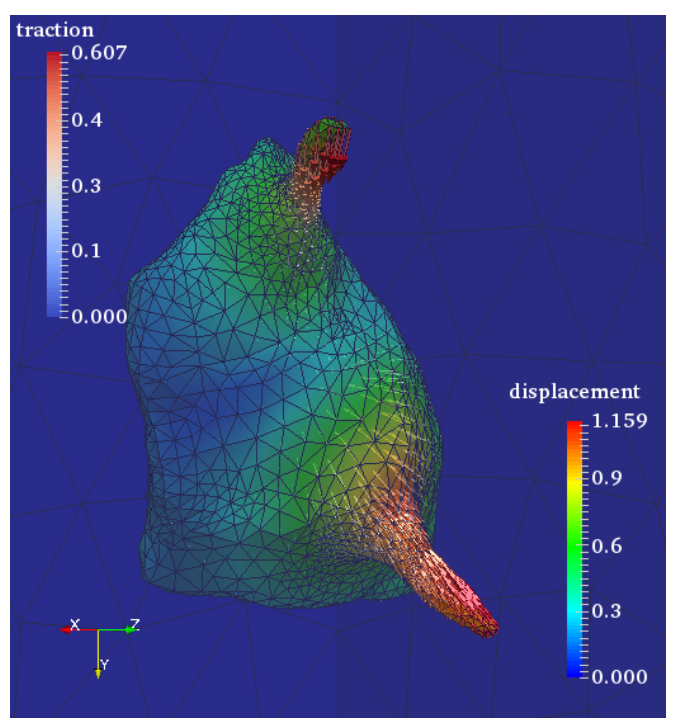

(a)

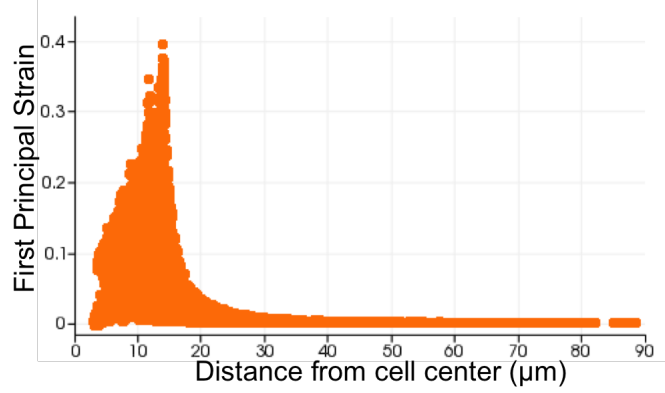

(c)

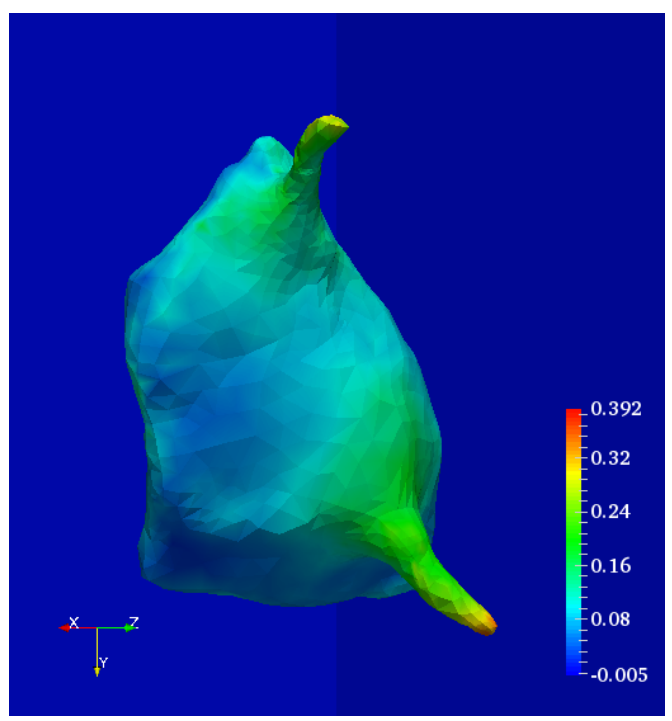

(b)

Figure 5: Results of the simulated forward problem for the microglial cell model on the deformed surface with $\gamma=$ 5. (a) Arrows display the traction field (in $\mathrm{kPa}$ ), and the deformed surface is colored by the displacement magnitude in $\mu \mathrm{m}$. (b) The first principal component of the Green-Lagrange strain tensor. (c) Distribution of the strain at the bead locations as a function of distance from the center. 


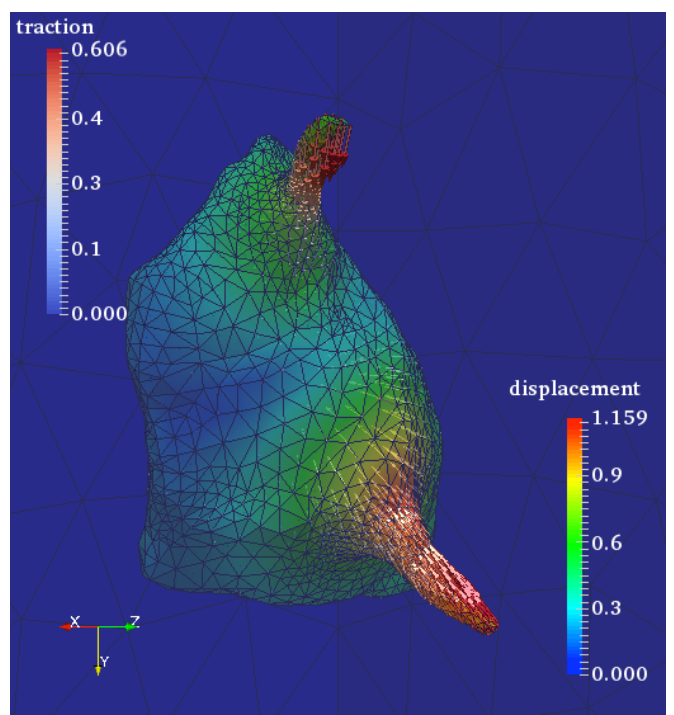

(a)

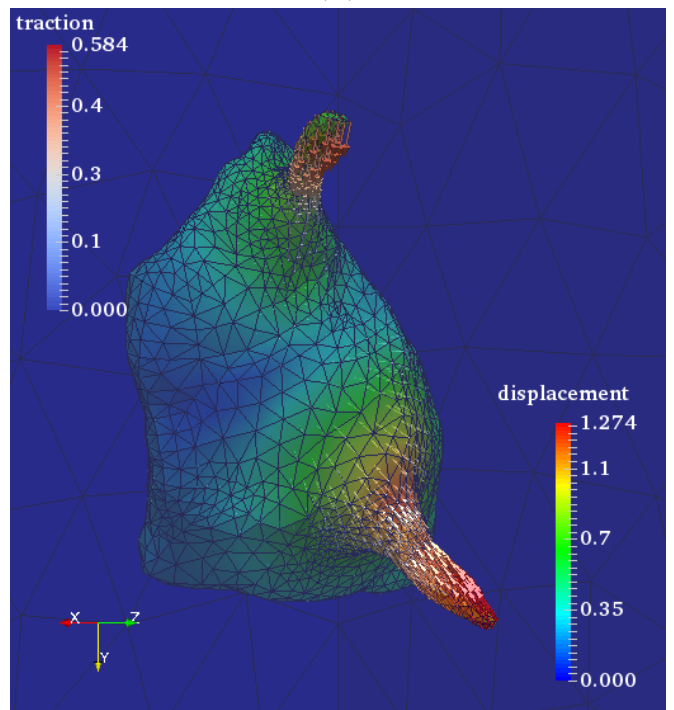

(c)

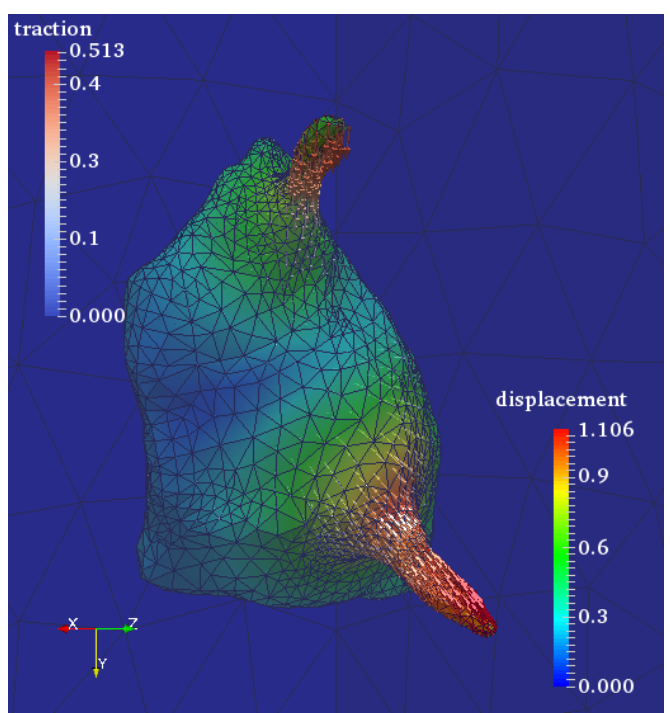

(b)

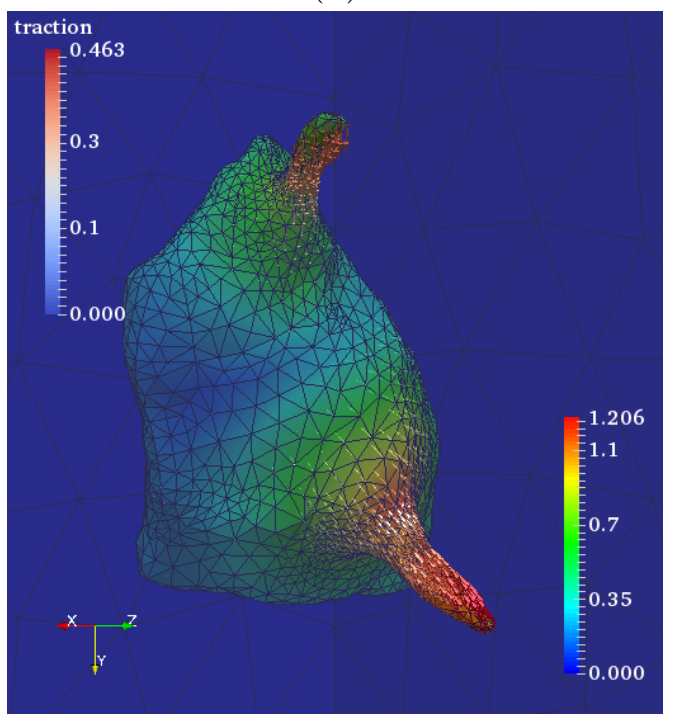

(d)

Figure 6: Results of the inverse analysis for the microglial cell on the deformed surface with $\gamma=5$. Arrows display the recovered traction field (in $\mathrm{kPa}$ ), and the deformed surface is colored by the recovered displacement magnitude in $\mu \mathrm{m}$. (a) Solution with nonlinear effects and $0 \%$ noise. (b) Solution with nonlinear effects and $1 \%$ noise. (c) Solution without nonlinear effects and $0 \%$ noise. (d) Solution without nonlinear effects and $1 \%$ noise. 


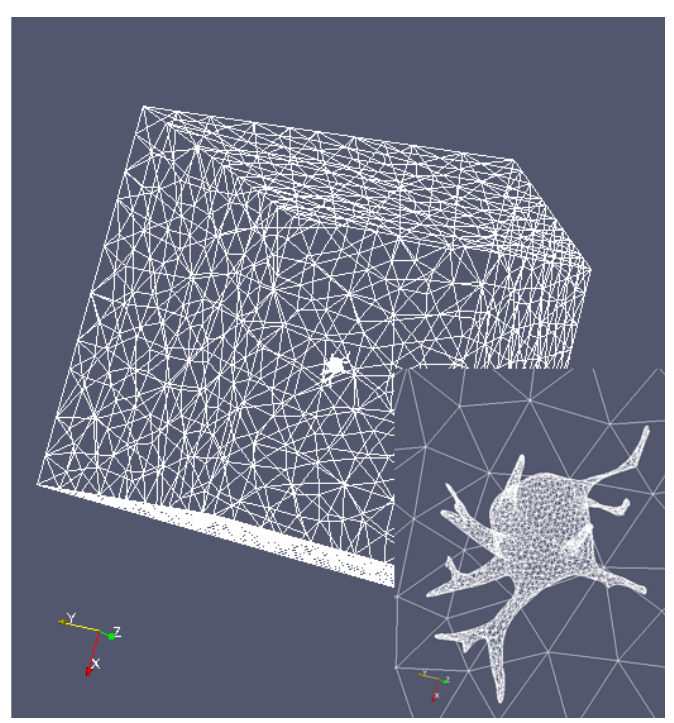

(a)

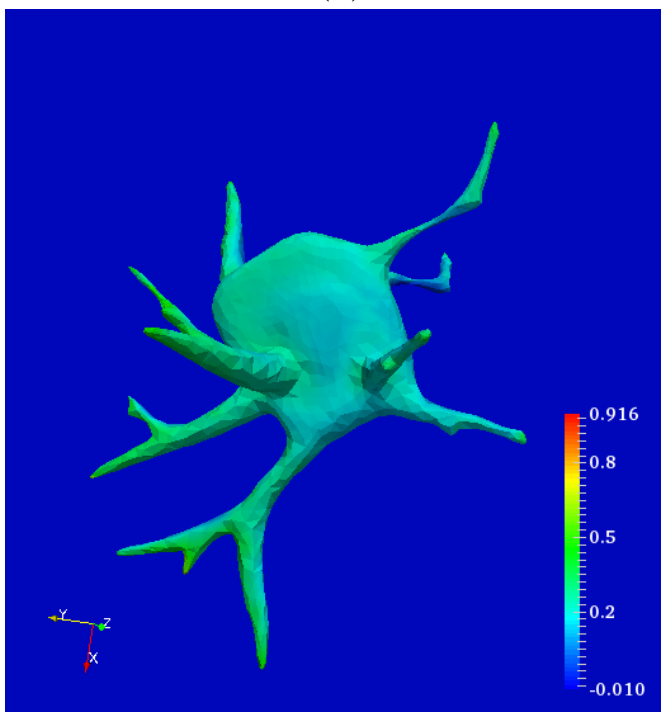

(c)

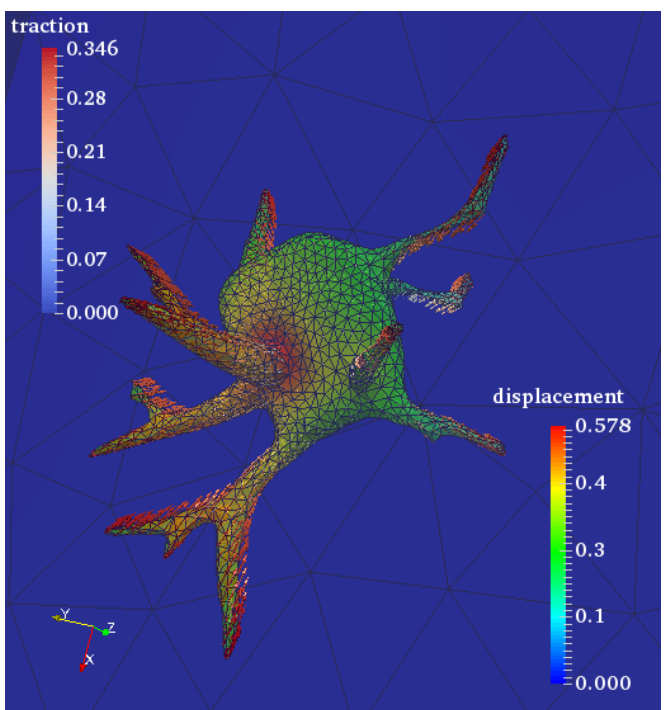

(b)

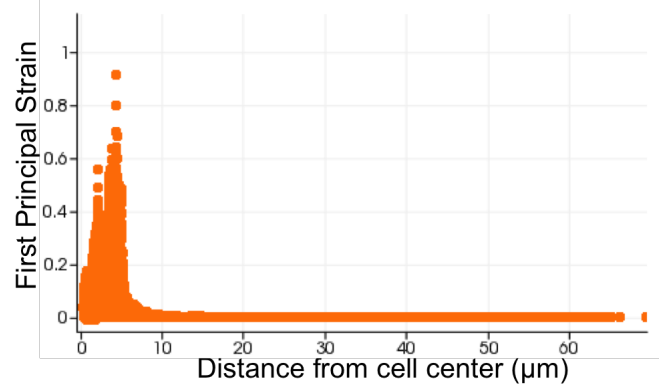

(d)

Figure 7: Neuronal cell: (a) Mesh on the exterior surface and the cell-matrix interface. Results of the simulated forward problem on the deformed surface with $\gamma=1$. (b) Arrows display the traction field (in $\mathrm{kPa}$ ), and the deformed surface is colored by the displacement magnitude in $\mu \mathrm{m}$. (c) The first principal component of the Green-Lagrange strain tensor. (d) Distribution of the strain at the bead locations as a function of distance from the center. 


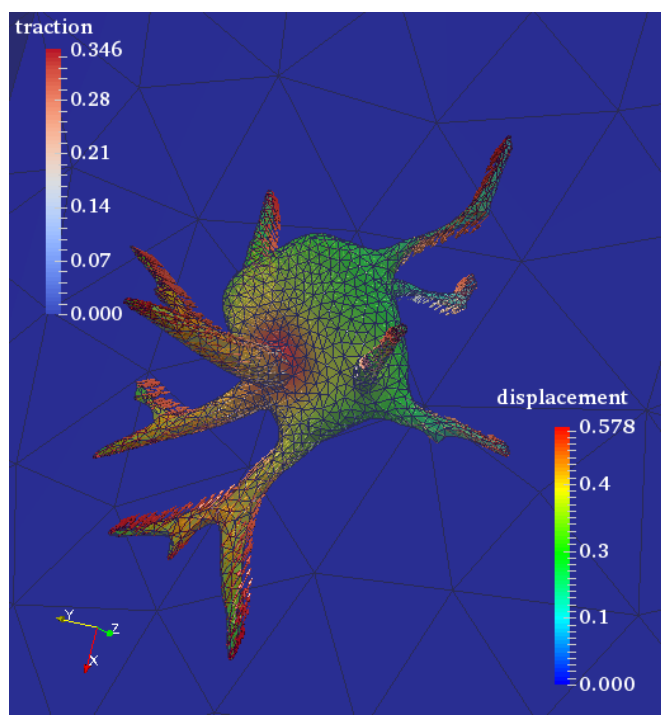

(a)

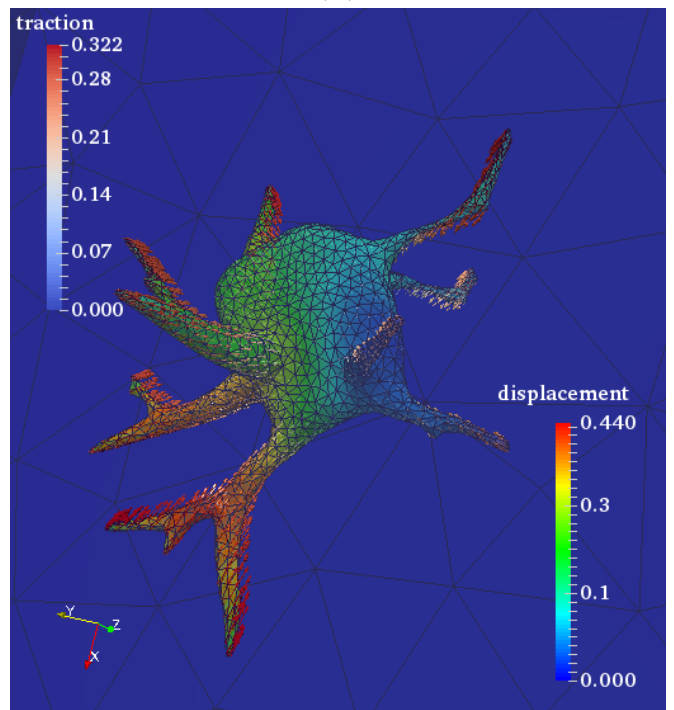

(c)

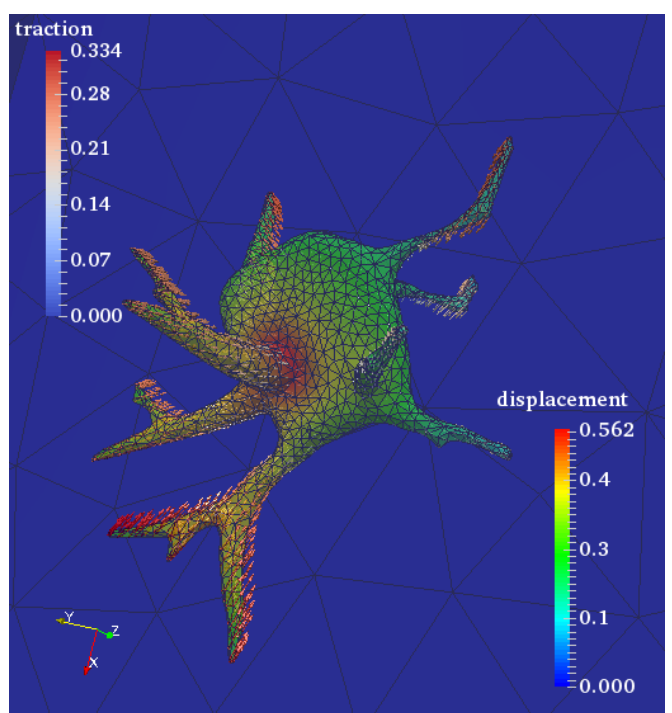

(b)

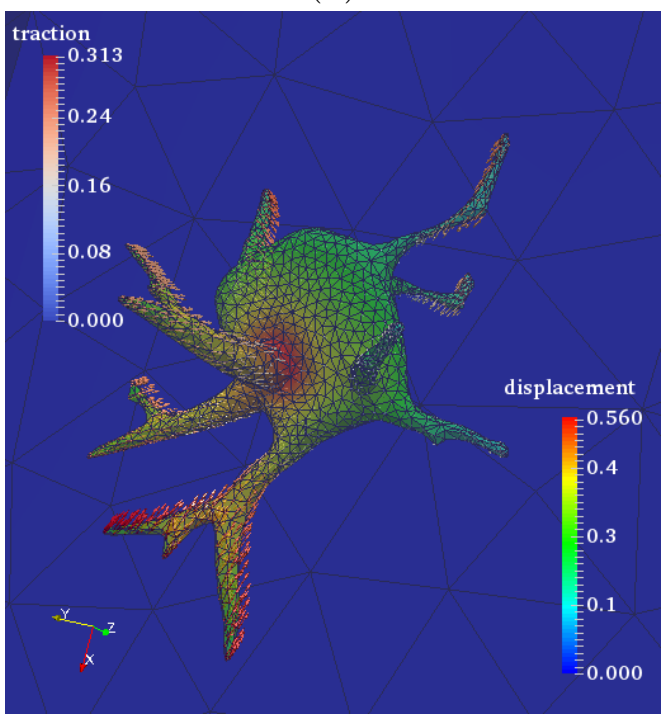

(d)

Figure 8: Results of the inverse analysis for the neuronal cell on the deformed surface with $\gamma=1$. Arrows display the recovered traction field (in $\mathrm{kPa}$ ), and the deformed surface is colored by the recovered displacement magnitude in $\mu \mathrm{m}$. (a) Solution with nonlinear effects and $0 \%$ noise. (b) Solution with nonlinear effects and $1 \%$ noise. (c) Solution without nonlinear effects and $0 \%$ noise. (d) Solution without nonlinear effects and $1 \%$ noise. 


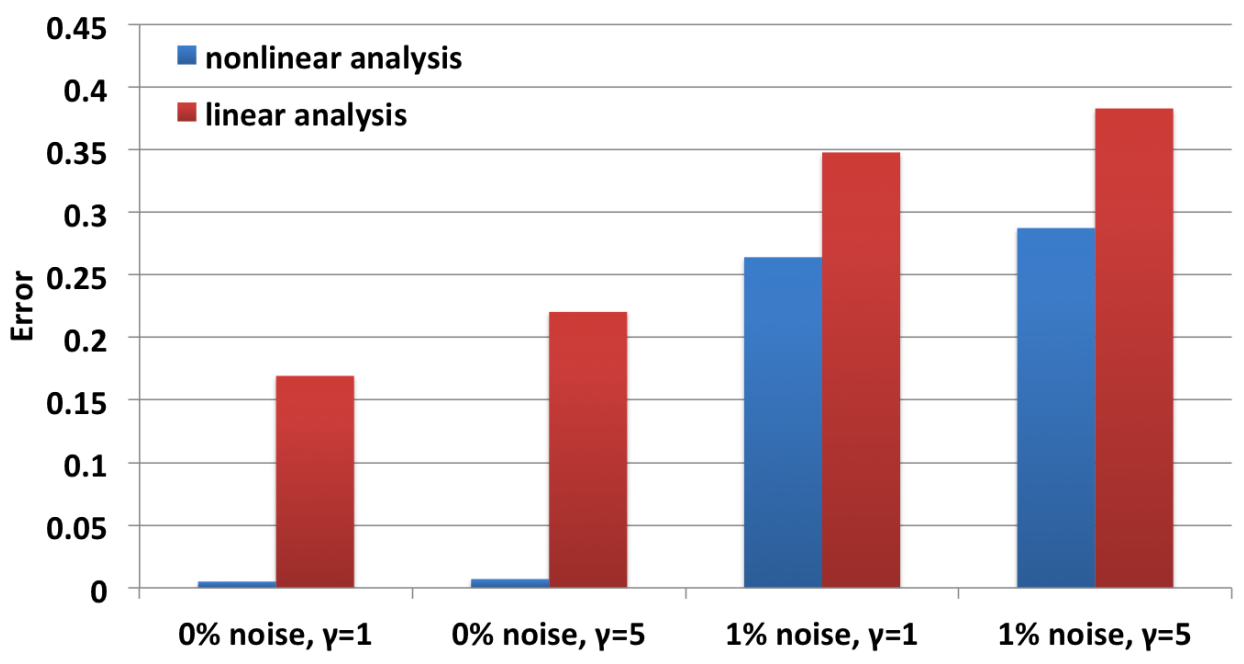

Figure 9: Error summary for the neuron cell model. 


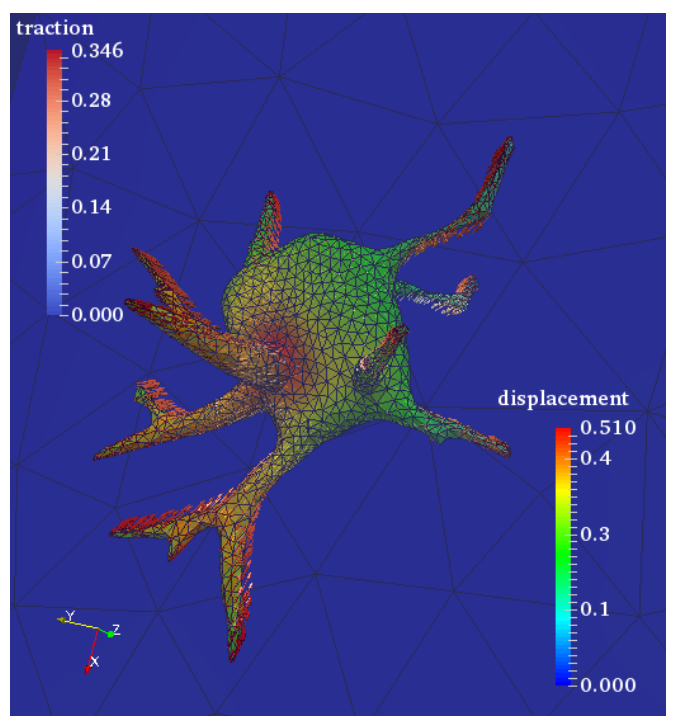

(a)

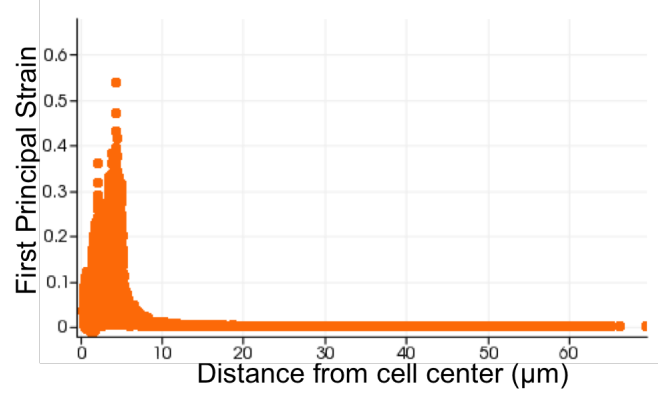

(c)

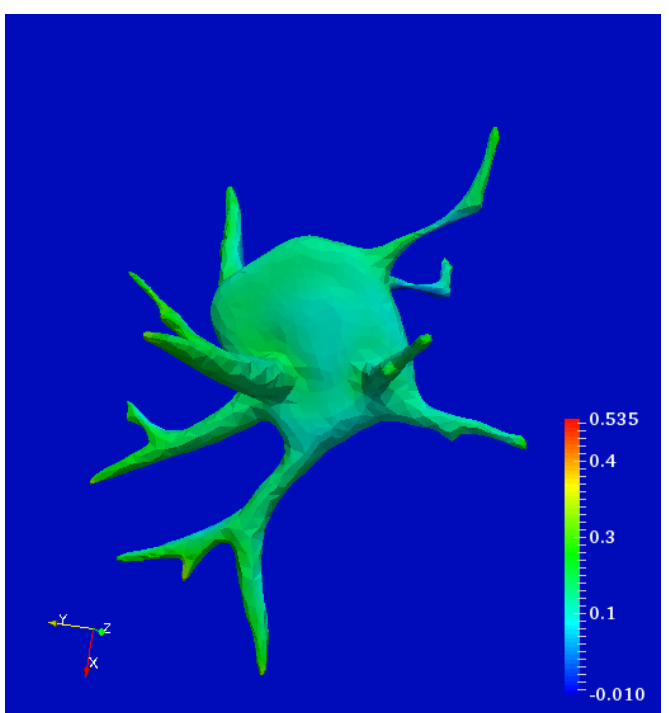

(b)

Figure 10: Results of the simulated forward problem for the neuronal cell model on the deformed surface with $\gamma=5$. (a) Arrows display the traction field (in $\mathrm{kPa}$ ), and the deformed surface is colored by the displacement magnitude in $\mu \mathrm{m}$. (b) The first principal component of the Green-Lagrange strain tensor. (c) Distribution of the strain at the bead locations as a function of distance from the center. 


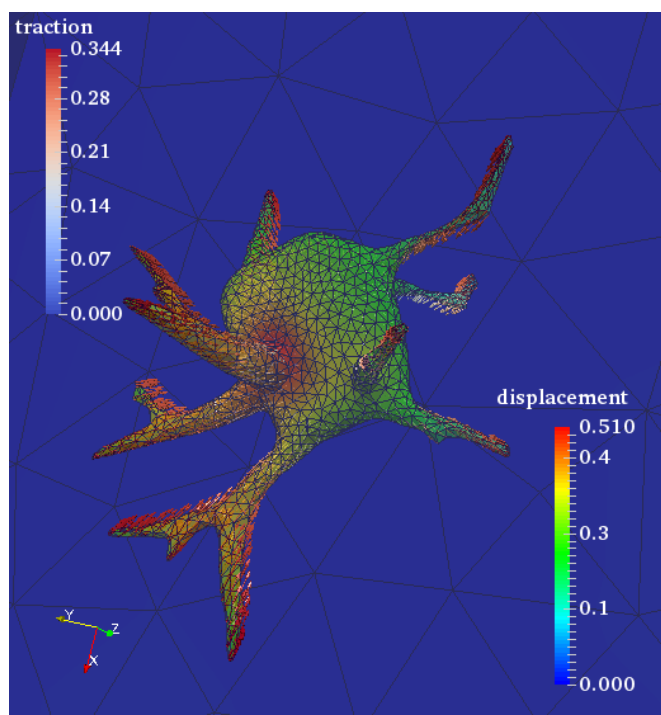

(a)

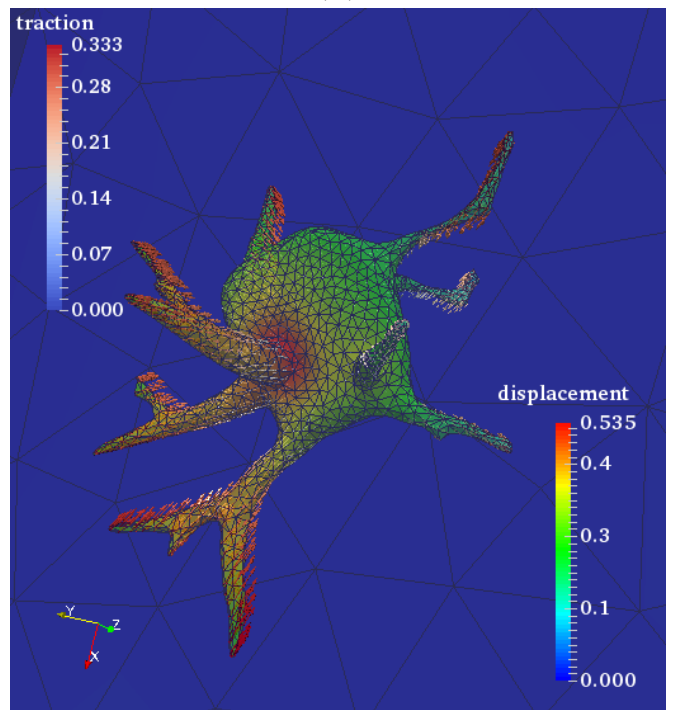

(c)

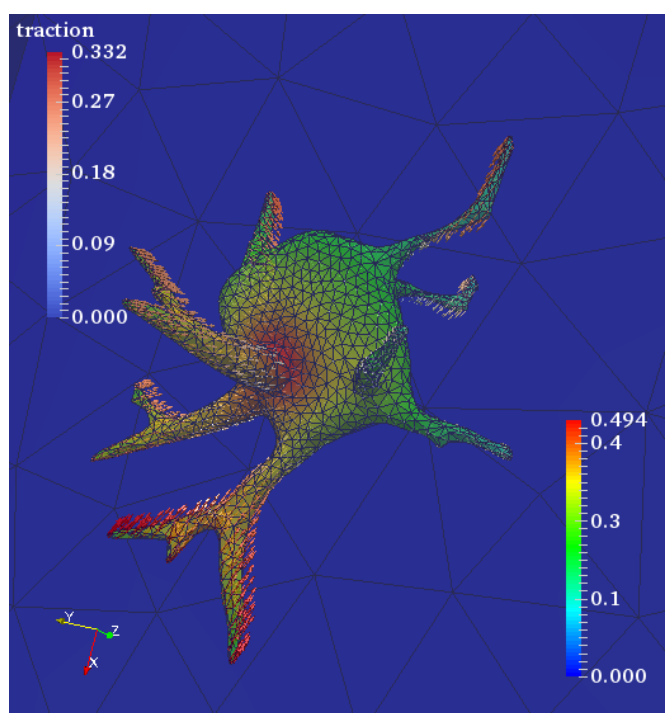

(b)

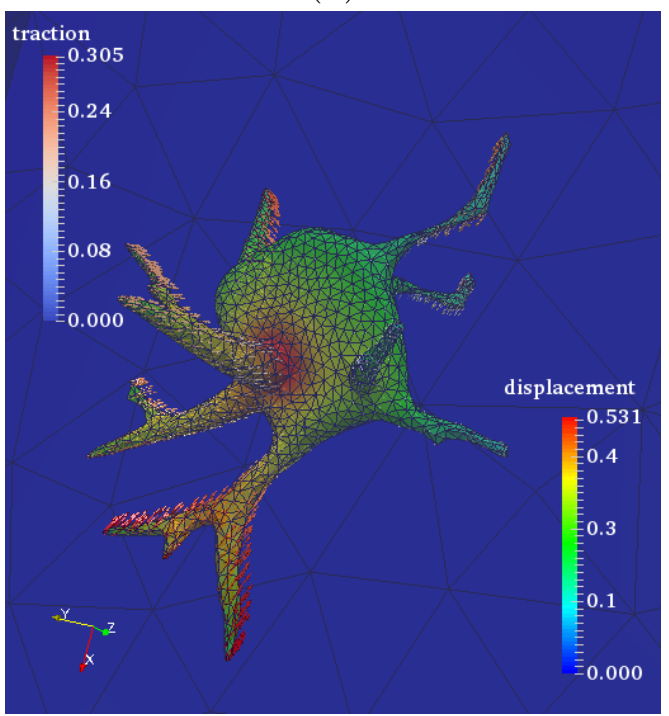

(d)

Figure 11: Results of the inverse analysis for the neuronal cell on the deformed surface with $\gamma=5$. Arrows display the recovered traction field (in $\mathrm{kPa}$ ), and the deformed surface is colored by the recovered displacement magnitude in $\mu \mathrm{m}$. (a) Solution with nonlinear effects and $0 \%$ noise. (b) Solution with nonlinear effects and $1 \%$ noise. (c) Solution without nonlinear effects and $0 \%$ noise. (d) Solution without nonlinear effects and $1 \%$ noise. 